\title{
Local Regularity for the Harmonic Map and Yang-Mills Heat Flows
}

\author{
Ahmad Afuni ${ }^{1}$ (D) \\ Received: 17 February 2019 / Accepted: 4 February 2021 / Published online: 2 March 2021 \\ (C) The Author(s) 2021
}

\begin{abstract}
We establish new local regularity results for the harmonic map and Yang-Mills heat flows on Riemannian manifolds of dimension greater than 2 and 4, respectively, obtaining criteria for the smooth local extensibility of these flows. As a corollary, we obtain new characterisations of singularity formation and use this to obtain a local estimate on the Hausdorff measure of the singular sets of these flows at the first singular time. Finally, we show that smooth blow-ups at rapidly forming singularities of these flows are necessarily nontrivial and admit a positive lower bound on their heat ball energies. These results crucially depend on some local monotonicity formulæ for these flows recently established by Ecker (Calc Var Partial Differ Equ 23(1):67-81, 2005) and the Afuni (Calc Var 555(1):1-14, 2016; Adv Calc Var 12(2):135-156, 2019).
\end{abstract}

Keywords Local regularity - Geometric heat flows · Harmonic map heat flow · Yang-Mills heat flow

Mathematics Subject Classification 35B65 $\cdot 35 \mathrm{~K} 55 \cdot 53 \mathrm{C} 07 \cdot 53 \mathrm{C} 44 \cdot 58 \mathrm{~J} 35$

\section{Introduction}

The main result of this paper is the following local regularity theorem for the harmonic map and Yang-Mills heat flows on Riemannian manifolds (see Sect. 2 for the setup):

Theorem A Let $(M, g)$ be a Riemannian manifold of dimension $n>2 k, \Omega \Subset M$, $c_{n, k}=\max \left\{\frac{1}{\sqrt{4 \pi}}, \sqrt{\frac{n-2 k}{2 \pi e}}\right\}$ and $\left.i_{0} \in\right] 0, \min \left\{\operatorname{inj}_{\Omega}, \pi /\left(2 \sqrt{\kappa_{\infty}^{+}}\right)\right\}\left[\right.$fixed, $\kappa_{\infty}$ being an upper bound on the sectional curvatures of $(\Omega, g)$. Then there exist geometric constants $\varepsilon, C>0$ such that if $\left.u: Q_{R}(X, T) \subset \Omega \times\right] 0, T\left[\rightarrow E \otimes \Lambda^{k-1} T^{*} M\right.$ is a harmonic

Ahmad Afuni

afuni@math.fu-berlin.de

1 Institut für Mathematik, Freie Universität Berlin, Arnimallee 3, 14195 Berlin, Germany 
map $(k=1)$ or Yang-Mills $(k=2)$ heat flow, then for any $R \leq i_{0}$, the implication

$$
\sup _{(x, t) \in Q_{R / 2}(X, T)} I_{k}\left(u, g ; x, t, \frac{R}{2 c_{n, k}}\right)<\varepsilon \Rightarrow R^{2 k} \sup _{Q_{R / 4}(X, T)} \frac{1}{2}|\psi|^{2} \leq C
$$

holds, where $\psi$ is the differential of $u$ or the curvature two-form, respectively, $Q_{R}(X, T)$ denotes the parabolic cylinder of radius $R$ centred at $(X, T)$, and $I_{k}\left(u, g ; x, t, \frac{R}{2 c_{n, k}}\right)$ is the heat ball energy (3.1) associated with $u$ over the heat ball $\frac{E_{\frac{R}{n-2 k}}^{n-2 k}}{2 c_{n, k}}(x, t)$.

The harmonic map heat flow was first introduced by Eells and Sampson [16] to smoothly deform smooth maps $\left(M^{n}, g\right) \rightarrow N \hookrightarrow \mathbb{R}^{K}$ between Riemannian manifolds $M$ and $N$ into harmonic ones. Key to their work was the fact that the target manifold $N$ had nonpositive sectional curvatures. Without this condition, the harmonic map heat flow does not necessarily exist for all time, which was shown by Coron and Ghidaglia [10] in the case where $n \geq 3$ and Chang et al. [7] in the case where $n=2$. Given that singularities are inevitable, one might ask how big the set of singularities - i.e. the singular set - is at the maximal time of a smooth harmonic map heat flow. This question was first answered by Struwe [34] in the case of compact $M$ of dimension 2 and arbitrary compact $N$, where the singular set was shown to consist of at most finitely many points. It was later shown by Grayson and Hamilton [20] in the case of compact $M$ of dimension at least 3 and compact $N$ (and implied by the work of Struwe [35] for Euclidean $M$ under suitable global restrictions on $u$ ) that the singular set is of codimension at least 2; moreover, they established the existence and nontriviality of smooth blow-ups of rapidly forming singularities. The crucial quantity in their analysis was a weighted scale-invariant energy of the form

$$
\int_{M} \frac{1}{2}|\psi|^{2}(\cdot, t) \cdot \Phi_{(X, T)}^{k}(\cdot, t)
$$

where $\Phi_{(X, T)}^{k}(x, t)=(T-t)^{k} \cdot \Phi_{(X, T)}$ for fixed $(X, T) \in M \times \mathbb{R}$ with $\Phi_{(X, T)}$ the canonical backward heat kernel on $M$ with singularity at $(X, T), \psi$ is the differential of $u$ and $k=1$. In particular, they showed that if (1.1) is smaller than a geometric constant $\varepsilon>0$ close to the maximal time $T$ for some fixed $X$, then a supremum bound on the differential of $u$ holds on a parabolic cylinder centred at $(X, T)$ (see Sect. 2 for the definition), which in turn implies that $u$ may be smoothly extended up to the maximal time $T$ in a neighbourhood of $X$; from this result, a characterisation of the singular set of $u$ at the maximal time is given in terms of a positive lower bound on (1.1) close to $T$, which then leads to an estimate on the $(n-2)$-dimensional Hausdorff measure of the singular set, as well as a strictly positive lower bound on the weighted energy (1.1) of smooth blow-ups at rapidly forming singularities. Ultimately, all of these results rely upon the scale invariance and monotonicity properties of (1.1), which were established by Struwe [35] and Hamilton [22].

The Yang-Mills heat flow of connections on a principal $G$-bundle over $\left(M^{n}, g\right)$ was introduced by Atiyah and Bott [4] to study the Morse theory of Yang-Mills 
connections and later exploited by Donaldson [11] to establish an equivalence between the stability of holomorphic vector bundles over Kähler manifolds and the existence of a unique Hermitian-Einstein connection. It has been shown by Råde [31] that given smooth initial data, this flow exists for all time and converges to a smooth Yang-Mills connection for compact $M$ of dimensions 2 and 3 . In dimension 4, it has recently been shown by Waldron that in stark contrast to the case of the harmonic map heat flow, singularities cannot occur in finite time [39], though convergence might only occur away from a singular set of finitely many points, as established by Struwe [36]. In higher dimensions, it has been shown that the Yang-Mills heat flow tends to develop singularities in finite time; this was done by Naito [25] in the case where $M$ is spherical and Grotowski [21] in the case where $M$ is Euclidean. It has likewise been shown that the singular set is of codimension at least 4 in the case of compact $M$ of dimension at least 5 by Chen et al. [9]; moreover, an analysis of rapidly forming singularities was carried out by Weinkove [41] in the higher-dimensional case. The key ingredient here is also a weighted energy of the form (1.1) with $\psi$ equal to the curvature two-form of the flow of connections and $k=2$, which again leads to an $\varepsilon$-regularity result as with the harmonic map heat flow from which an estimate on the singular set at the maximal time as well as a lower bound on the weighted energy of smooth blow-ups at rapidly forming singularities readily follows. Again, these results rely upon scale invariance and monotonicity properties of (1.1) established by Chen and Shen [8] and Hamilton [22].

Theorem A is an analogue of both of these $\varepsilon$-regularity results; however, in contrast to the quantity (1.1), ours, which is expressed as a supremum of a collection of socalled heat ball energies, depends only on local data, as these heat balls completely lie within a parabolic cylinder with proportional radius centred at the same point, which allows us to state a criterion for the local extensibility of $u$ up to the maximal time $T$ valid on Riemannian manifolds without imposing any additional conditions on $M$ or $u$. While it is possible to introduce a cut-off function in (1.1) to prove an analogue of Theorem A for radii smaller than a constant depending on geometry and the local energy of $u$ as has been done in [23] for the Yang-Mills-Higgs flow, our approach works out more cleanly and closely parallels the analogous regularity results in the static case, which are stated in terms of suitable rescaled energies on balls (see [32, Proposition 2.4] and [32, Theorem 3.1] for the case of harmonic maps as well as [30, Theorem 1] and [26, Lemma 3.1] for the case of Yang-Mills connections). Furthermore, our proof rests on a fairly simple blow-up argument as well as suitable local monotonicity formulæ established by Ecker [14] for the harmonic map heat flow on Euclidean domains and the author [1,3] more generally for both the harmonic map and Yang-Mills heat flows on Riemannian manifolds. The blow-up argument we use was employed by White [42] in establishing a similar local regularity result for the mean curvature flow (see also [13, Chap. 5]), which ultimately motivated our approach here.

Theorem A likewise gives rise to a characterisation of the singular set $\mathcal{S}$ of $u$ at the maximal time, as well as a local estimate on its Hausdorff measure under the assumption of summability of $|\psi|^{2}$ on $\Omega \times[0, T$, which is the content of the following theorem. 
Theorem B Let $\psi$ be as in Theorem A and suppose $|\psi|^{2} \in L^{1}(\Omega \times[0, T[)$. Then the singular set $\mathcal{S}$ is closed and for any $K \Subset \Omega$ and $\left.\delta_{0} \in\right] 0$, dist $(K, \partial \Omega)[$, the estimate

$$
\mathscr{H}^{n-2 k}(\mathcal{S} \cap K) \leq \varepsilon_{1} \limsup _{t \nearrow T} \int_{B_{\delta_{0}}(\mathcal{S} \cap K)} \frac{1}{2}|\psi|^{2}(\cdot, t) d v o l_{g}
$$

holds, where $\varepsilon_{1}>0$ is a geometric constant and $\mathscr{H}^{n-2 k}$ denotes the $(n-2 k)$ dimensional Hausdorff measure on $M$.

As another corollary of Theorem A, we obtain the following nontriviality result for smooth blow-ups at rapidly forming singularities in the form of a positive lower bound on the heat ball energy of the blow-up.

Theorem $\mathrm{C}$ Let $u$ and $\psi$ be as in Theorem A and suppose $u$ has a rapidly forming singularity at $(X, T)$ in the sense that

$$
\sup _{(x, t) \in Q_{R_{0}}(X, T)}(T-t)^{k} \frac{1}{2}|\psi|^{2}(x, t) \leq C_{0}
$$

holds for some $C_{0}>0$ and $R_{0}>0$. Then $u$ admits a sequence of rescalings converging locally uniformly in $C^{\infty}$ (modulo gauge) to a smooth self-similar harmonic map or Yang-Mills heat flow $u_{\infty}$ on $\left.\mathbb{R}^{n} \times\right]-\infty, 0[$ satisfying the estimate

$$
I_{k}\left(u_{\infty}, g_{\delta} ; 0,0, R\right) \geq \varepsilon
$$

for all $R>0$, where $\varepsilon$ is as in Theorem A and $g_{\delta}$ denotes the Euclidean metric on $\mathbb{R}^{n}$.

We note that an analogue of Theorem A has been established for the Ricci flow by Ni [27], though in that case its application to the study of singularities is far more subtle and has only been carried out in the case of rapidly forming singularities (see [17]).

The structure of this paper is as follows. In Sect. 2 we describe the underlying geometric setup and introduce the harmonic map and Yang-Mills heat flows, as well as some of their important properties, stating well-known facts in the context of our considerations, as well as the local monotonicity formulæ of these flows on heat balls which shall subsequently play an important rôle. In Sect. 3, we prove Theorem A and show how the $\varepsilon$-regularity condition yields a necessary and sufficient condition for the local smooth extensibility of these flows up to the maximal time; in the process, we obtain an alternative local regularity theorem based around parabolic cylinders rather than heat balls. In Sect. 4 the singular set at the maximal times of these flows is defined and more explicitly described in terms of the results of Sect. 3, which then leads to a proof of Theorem B. Finally, in Sect. 5, we turn our attention to rapidly forming singularities of these flows and show that they admit smooth, nontrivial blow-ups, culminating in a proof of Theorem $\mathrm{C}$. Throughout this paper, we have attempted to treat both the Yang-Mills and harmonic map heat flows simultaneously, abstracting away their common properties. The possibility of this approach suggests that there might be a more general principle at play. 


\section{Setup}

\subsection{Geometry}

Throughout this paper we will be dealing with a Riemannian manifold $\left(M^{n}, g\right)$ of dimension $n>2 k>0$ with $k$ to be fixed shortly according to the heat flow under consideration. We shall adopt the notation of [40] and [28] for all Riemannian geometric quantities, operators and spaces. We shall write $\langle\cdot, \cdot\rangle$ and $|\cdot|$ for the inner product and norm associated with $g$ respectively and shall append the metric as a subscript when ambiguity must be avoided. Furthermore, when considering tensor products (and exterior products) of bundles constructed from the tangent bundle $T M$ (or the cotangent bundle $T^{*} M$ ) and Riemannian vector bundles, we shall simply write $\langle\cdot, \cdot\rangle$ and $|\cdot|$ for the naturally induced inner product and norm, and given (families of) connections $\nabla$ on these bundles, we shall again write $\nabla$ for the induced connections on these constructed bundles. We shall write $(\cdot, \cdot)$ for the canonical fibrewise bilinear pairings of elements of $E \otimes \otimes T^{*} M$ with $\otimes T M$ as well as $E \otimes \Lambda T^{*} M$ with $\Lambda T M$, where $E$ is any vector bundle, and the fibrewise interior product of a vector field $X$ with a section $\alpha$ of $E \otimes \Lambda T^{*} M$ shall be denoted by $X\llcorner\alpha$. The Euclidean metric on $\mathbb{R}^{n}$ and its subsets shall be denoted by $g_{\delta}$.

In all of our considerations, $\Omega$ will denote a fixed, relatively compact subset of $M$. Thus, we may find constants $i_{0}>0$ and $\kappa_{-\infty}, \kappa_{\infty}>0$ such that $\operatorname{inj}_{\Omega}>i_{0}$ and the sectional curvature bounds

$$
-\kappa_{-\infty} \leq \sec \leq \kappa_{\infty}
$$

hold on $\Omega$. For simplicity, we will assume that $i_{0}<\frac{\pi}{2 \sqrt{\kappa_{\infty}}}$ if $\kappa_{\infty}>0$. Note that for fixed $x \in \Omega$, we obtain the estimate

$$
\Lambda_{-\infty}\left(\frac{1}{2} \mathfrak{r}(\cdot, t)^{2}\right) g^{\mathfrak{r}}(\cdot, x) \leq g-\nabla^{2}\left(\frac{1}{2} \mathfrak{r}(\cdot, x)^{2}\right) \leq \Lambda_{\infty}\left(\frac{1}{2} \mathfrak{r}(\cdot, x)^{2}\right) g^{\mathfrak{r}}(\cdot, x)
$$

in $\Omega \cap B_{i_{0}}(x)$, where $\mathfrak{r}(\cdot, x)$ denotes the distance function measured from $x$,

$$
g^{\mathfrak{r}}=g-\mathrm{dr}(\cdot, x) \otimes \mathrm{d} \mathfrak{r}(\cdot, x)
$$

and $\Lambda_{ \pm \infty}$ are constants which may be determined explicitly in terms of $\kappa_{ \pm}$by means of a Hessian comparison theorem (cf. [28, Theorem 27, p.175]).

For $x \in M$ and $r>0$, we denote the geodesic ball of radius $r$ centred at $x$ by $B_{r}(x):=\{\mathfrak{r}(\cdot, x)<r\}$ and for $\left.\left.t \in\right] 0, T\right]$, we introduce the parabolic cylinder of radius $r$ centred at $(x, t)$ as

$$
\left.Q_{r}(x, t):=B_{r}(x) \times\right] t-r^{2}, t[.
$$


For later purposes, we introduce for each $\lambda>0$ and $X \in M$ the (crudely) rescaled metric $g_{\lambda}^{X}: B_{\text {inj }_{X} / \lambda}(0) \subset \mathbb{R}^{n} \rightarrow T^{*} \mathbb{R}^{n} \otimes T^{*} \mathbb{R}^{n}$ defined by

$$
g_{\lambda}^{X}(y)=\sum_{i, j=1}^{n} g_{i j}(\lambda y) \mathrm{d} y^{i} \otimes \mathrm{d} y^{j}
$$

where $\left\{g_{i j}\right\}$ are the components of $g$ in geodesic normal coördinates about $X$ and $\left\{y^{i}\right\}$ denote Euclidean coördinates.

We shall write $\sqrt{g}$ for the volume density associated with $g$ and some fixed coördinate system which shall be understood from context. Moreover, the associated volume measure on $(M, g)$ shall be written $\mathrm{dvol}_{g}$. Integrals over space-time regions shall be written in double integral notation and, when there is no possibility of confusion, we shall not append a measure to the integrand.

\subsection{Flows}

We shall now proceed to describe the flows we are interested in. In both cases, we have a Riemannian vector bundle $V \rightarrow E \rightarrow M$ with fibrewise inner product $\langle\cdot, \cdot\rangle$, a one-parameter family of connections $\nabla$, and a one-parameter family of $E$-valued $(k-1)$-forms

$$
\left\{u(\cdot, t): M \rightarrow E \otimes \Lambda^{k-1} T^{*} M\right\}_{t \in[0, T[}
$$

with a distinguished fundamental $k$-form $\left\{\psi(\cdot, t): M \rightarrow E \otimes \Lambda^{k} T^{*} M\right\}_{t \in[0, T[\text { satis- }}$ fying an equation of the form

$$
\partial_{t} \psi-\Delta \psi=B
$$

where $\Delta$ denotes the connection Laplacian associated with the family of connections on $E$ and the Levi-Cività connection on $T M$, and $B$ is a suitable polynomial expression in $\psi$ and $\nabla \psi$.

\subsubsection{Harmonic Map Heat Flow}

The harmonic map heat flow is given by a one-parameter family of smooth maps

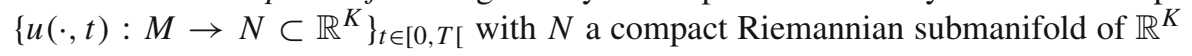
such that the equation

$$
\left(\partial_{t}-\Delta_{g}\right) u=-\sum_{i, j} g^{i j}\left(b \circ u, \partial_{i} u \otimes \partial_{j} u\right)
$$

holds, where $b$ denotes the second fundamental form of $N$ extended to an $\left(\mathbb{R}^{K}\right)^{*} \otimes$ $\left(\mathbb{R}^{K}\right)^{*}$-valued mapping by setting it equal to zero when paired with vectors normal 
to $N$. For this flow, $k=1, \psi=\mathrm{d} u, V=\mathbb{R}^{K}, E=\mathbb{R}^{K}$, the trivial vector bundle with standard fibre $\mathbb{R}^{K}$, and $\nabla$ is the canonical trivial connection on $E$. Moreover, by differentiating (2.4) and carefully interchanging derivatives (or equivalently employing a Weizenböck-type argument [29, Theorem 4.22]), we see that $\mathrm{d} u$ satisfies an equation of the form (2.3) with

$$
\begin{aligned}
B= & -\sum_{i, j=1}^{n} g^{i j}\left(\nabla b \circ u, \partial_{i} u \otimes \partial_{j} u \otimes \partial_{k} u\right) \otimes \mathrm{d} x^{k} \\
& -\sum_{i, j=1}^{n} 2\left(b \circ u,\left(\nabla \mathrm{d} u, \partial_{k} \otimes \partial_{i}\right) \otimes \partial_{j} u\right) \otimes \mathrm{d} x^{k} \\
& -\sum_{i, j, k=1}^{n} R_{i j} g^{i k} \partial_{k} u \otimes \mathrm{d} x^{j},
\end{aligned}
$$

where $\nabla b$ is the covariant differential of the second fundamental form of $N$ extended as above to an $\bigotimes_{i=1}^{3}\left(\mathbb{R}^{K}\right)^{*}$-valued mapping, its last slot corresponding to the direction of covariant differentiation, and $\left\{R_{i j}\right\}$ are the components of the Ricci curvature tensor of $g$.

Given a harmonic map heat flow $u$, we may rescale it as follows: Let $\vartheta_{x}: B_{\text {inj }_{x}}(0) \subset$ $\mathbb{R}^{n} \rightarrow B_{\text {inj }_{x}}(x) \subset M$ denote a geodesic normal parametrisation centred at $x \in M$ (fixed once and for all). Defining

$$
u_{\lambda}^{(x, t)}(y, s):=\left(\vartheta_{x}^{*} u\right)\left(\lambda y, t+\lambda^{2} s\right)=u\left(\vartheta_{x}(\lambda y), t+\lambda^{2} s\right),
$$

we obtain a one-parameter family of maps

$$
\left\{u_{\lambda}^{(x, t)}(\cdot, s): B_{\operatorname{inj}_{x} / \lambda}(0) \rightarrow N\right\}_{s \in]-\frac{t}{\lambda^{2}}, \frac{T-t}{\lambda^{2}}[}
$$

solving the equation (2.4) with respect to the metric $g_{\lambda}^{x}$. In the case where $(M, g)$ is Euclidean space, we say that $u$ is self-similar about $(x, t)$ if $u(y, s)=u_{\lambda}^{(x, t)}(y, s)$ for all $\lambda>0$ and $(y, s) \in \mathbb{R}^{n} \times \mathbb{R}$ for which both sides are defined.

\subsubsection{Yang-Mills Heat Flow}

Let $G \rightarrow P \rightarrow M$ be a principal bundle with compact semi-simple Lie group $G$ as its structure group, and write $E_{\mathfrak{g}}$ for the vector bundle associated with $P$ and the adjoint representation of $G$ on its Lie algebra $\mathfrak{g}$. The (negative) Killing form on $\mathfrak{g}$ endows $E_{\mathfrak{g}}$ with the structure of a Riemannian vector bundle; moreover, any connection $\omega$ on $P$ induces a unique covariant derivative operator $\nabla$ on $E_{\mathfrak{g}}$ compatible with this Riemannian structure. The Yang-Mills heat flow is given by a one-parameter family of connections $\left\{\omega(\cdot, t): P \rightarrow \mathfrak{g} \otimes T^{*} P\right\}_{t \in[0, T[\text { such that the equation }}$

$$
\underline{\partial_{t} \omega}=\operatorname{div} \underline{\Omega}^{\omega}
$$


holds, where $\partial_{t} \omega(\cdot, t)$ is the unique section of $E_{\mathfrak{g}} \otimes T^{*} M$ having $\left.\partial_{t} \omega: P \times\right] 0, T[\rightarrow$ $\mathfrak{g} \otimes T^{*} P$ as its horizontal lift, $\underline{\Omega}^{\omega}: M \times\left[0, T\left[\rightarrow E_{\mathfrak{g}} \otimes \Lambda^{2} T^{*} M\right.\right.$ is the curvature twoform of $\omega$, and the divergence operator div is induced by the Levi-Cività connection on $T M$ and $\omega(\cdot, t)$. For background material on this setup in accordance with our viewpoint, we refer the reader to [6, IV.2].

In order to study $\{\omega(\cdot, t)\}$ on $M$, fix a connection $\omega_{0}$ on $P$. The difference $\omega(\cdot, t)-\omega_{0}$ is then a horizontal $G$-equivariant $\mathfrak{g}$-valued one-form on $P$ and therefore corresponds to a $E_{\mathfrak{g}}$-valued one-form $u(\cdot, t): M \rightarrow E_{\mathfrak{g}} \otimes T^{*} M$ for each $t$ which satisfies the equation $\partial_{t} u=\partial_{t} \omega$. Note that the choice of $\omega_{0}$ is not canonical. Thus, for this flow, $k=2, V=\mathfrak{g}, \overline{E=} E_{\mathfrak{g}}, u$ is as above, $\psi=\underline{\Omega}^{\omega}$ and $\nabla$ is the one-parameter family of connections on $E_{\mathfrak{g}}$ induced by $\omega$. Analogously to the case of the harmonic map heat flow, using the fact that

$$
\partial_{t} \underline{\Omega}^{\omega}=\mathrm{d}^{\nabla} \underline{\partial_{t} \omega}
$$

$\mathrm{d}^{\nabla}$ denoting the exterior covariant derivative associated with the connection on $E_{\mathfrak{g}}$ arising from $\omega$ and the Levi-Cività connection on $T M$ (cf. [29, 2.75]), differentiating (2.6) and employing a Weitzenböck-type argument, we deduce that $\underline{\Omega}^{\omega}$ satisfies an equation of the form (2.3) with

$$
\begin{aligned}
B= & -\sum_{i, j, k, p=1}^{n} g^{j k}\left[\left(\underline{\Omega}^{\omega}, \partial_{k} \wedge \partial_{i}\right),\left(\underline{\Omega}^{\omega}, \partial_{j} \wedge \partial_{p}\right)\right] \otimes \mathrm{d} x^{i} \wedge \mathrm{d} x^{p} \\
& -\sum_{i, j, k, l=1}^{n} g^{i l} R_{l j}\left(\underline{\Omega}^{\omega}, \partial_{i} \wedge \partial_{k}\right) \otimes \mathrm{d} x^{j} \wedge \mathrm{d} x^{k} \\
& +\sum_{i, j, k, l, m=1}^{n} g^{j k} R_{\cdot l i k}^{m}\left(\underline{\Omega}^{\omega}, \partial_{m} \wedge \partial_{j}\right) \otimes \mathrm{d} x^{i} \wedge \mathrm{d} x^{l},
\end{aligned}
$$

where $[\cdot, \cdot]$ is the fibrewise Lie bracket on $E_{\mathfrak{g}}$ naturally induced by that of $\mathfrak{g}$ and $\left\{R_{\cdot \text { lik }}^{m}\right\}$ are the components of the Riemann curvature tensor of $g$, where the sign convention is that of [37].

We now more explicitly describe $\omega(\cdot, t)$ locally. To this end, we fix a local section $\sigma: U \rightarrow P$ and let $\Psi_{\sigma}: U \times \mathfrak{g} \rightarrow E_{\mathfrak{g}}$ be the local bundle parametrisation induced by $\sigma$. Then $u(x, t)=\sum_{i=1}^{n} \Psi_{\sigma}\left(x, A_{i}(x, t)-\left(A_{0}\right)_{i}(x, t)\right) \otimes \mathrm{d} x^{i}$ for $(x, t) \in U \times[0, T[$, where

$$
A(\cdot, t):=\sum_{i=1}^{n} A_{i}(\cdot, t) \otimes \mathrm{d} x^{i}:=\sigma^{*} \omega(\cdot, t)
$$

is the local connection form and

$$
A_{0}:=\sum_{i=1}^{n}\left(A_{0}\right)_{i}(x) \otimes \mathrm{d} x^{i}:=\sigma^{*} \omega_{0} .
$$


The curvature two-form is then locally given as

$$
F(\cdot, t)=\sum_{i<j} F_{i j}(\cdot, t) \otimes \mathrm{d} x^{i} \wedge \mathrm{d} x^{j}: U \rightarrow \mathfrak{g} \otimes \Lambda^{2} T^{*} M
$$

with $F_{i j}=\partial_{i} A_{j}-\partial_{j} A_{i}+\left[A_{i}, A_{j}\right]$. We may therefore locally describe a Yang-Mills heat flow by means of a smooth one-parameter family of local $\mathfrak{g}$-valued one-forms $\{A(\cdot, t)\}_{t \in[0, T[}$ solving the system of equations

$$
\partial_{t} A_{i}=\sum_{p, q=1}^{n} g^{p q}\left(\partial_{p} F_{q i}+\left[A_{p}, F_{q i}\right]-\sum_{r=1}^{n}\left(\Gamma_{p q}^{r} F_{r i}+\Gamma_{p i}^{r} F_{q r}\right)\right)
$$

on $U \times] 0, T[$ for each $i \in\{1, \ldots, n\}$.

Similarly to the harmonic map heat flow, we may rescale a Yang-Mills heat flow as follows: Fix $(x, t) \in M \times] 0, T]$ and a local section $\sigma: B_{\text {inj }_{x}}(x) \rightarrow P$. As before, we let $\vartheta_{x}: B_{\operatorname{inj}_{x}}(0) \rightarrow B_{\operatorname{inj}_{x}}(x)$ denote a geodesic normal coördinate parametrisation. Defining

$$
A_{\lambda}^{(x, t)}(y, s):=\left(\vartheta_{x}^{*} A\right)\left(\lambda y, t+\lambda^{2} s\right)=\sum_{i=1}^{n} \lambda A_{i}\left(\vartheta_{x}(\lambda y), t+\lambda^{2} s\right) \otimes \mathrm{d} y^{i}
$$

we obtain a smooth one-parameter family

$$
\left\{A_{\lambda}^{(x, t)}(\cdot, s): B_{\operatorname{inj}_{x} / \lambda}(0) \rightarrow \mathfrak{g} \otimes T^{*} \mathbb{R}^{n}\right\}_{s \in]-\frac{t}{\lambda^{2}}, \frac{T-t}{\lambda^{2}}[}
$$

solving the system of equations (2.8) with respect to the metric $g_{\lambda}^{x}$. This then gives rise to a family of connections $\left\{u_{\lambda}^{(x, t)}(\cdot, s)\right\}_{s \in]-\frac{t}{\lambda^{2}}, \frac{T-t}{\lambda^{2}}[}$ on the trivial bundle $B_{\text {inj }_{x} / \lambda}(0) \times G$ evolving by the Yang-Mills heat flow, where the metric tensor on the base manifold is given by $g_{\lambda}^{x}$. As with the harmonic map heat flow, if $(M, g)$ is Euclidean, we call $u$ self-similar about $(x, t)$ if $A_{i}(y, s) \equiv\left(A_{\lambda}^{(x, t)}\right)_{i}(y, s)$ for all $\lambda>0, i \in\{1, \ldots, n\}$ and $(y, s) \in \mathbb{R}^{n} \times \mathbb{R}$ for which both sides are defined.

In contrast to the case of the harmonic map heat flow (with isometrically embedded target manifold), there is no preferred representation of a connection $\omega$ as an $E_{\mathfrak{g}}$-valued one-form; indeed, from a differential geometric point of view, connections are usually considered equivalent if they are related by the action of the gauge group: Given a connection $\omega$ on $P$ with local one-forms $\left\{A^{\sigma}=\sum_{i=1}^{n} A_{i}^{\sigma} \otimes \mathrm{d} x^{i}: \sigma\right.$ a local section of $\left.P\right\}$ and a section $g: U \rightarrow E_{G}$ of the bundle $G \rightarrow E_{G} \rightarrow M$ associated with $P$ and the action of $G$ on itself by conjugation, which we may locally write as

$$
g(x)=\Phi_{\sigma}\left(x, g^{\sigma}(x)\right)
$$


with $\sigma$ a local section of $P$ and $\Phi_{\sigma}$ the local bundle parametrisation of $E_{G}$ associated with $\sigma$, the collection of local one-forms

$$
\left\{\widetilde{A}^{\sigma}=\sum_{i=1}^{n} \widetilde{A}_{i}^{\sigma} \otimes \mathrm{d} x^{i}\right\}
$$

with $\widetilde{A}_{i}^{\sigma}:=\operatorname{Ad}_{g^{\sigma}}\left(A_{i}^{\sigma}\right)-\partial_{i} g^{\sigma} \cdot\left(g^{\sigma}\right)^{-1}$ gives rise to a connection $g \cdot \omega$ on $P$, where Ad denotes the adjoint representation of $G$ on $\mathfrak{g}$ and $\cdot$ the natural right action of $G$ on $T G$. The connection corresponding to $g \cdot \omega$ satisfies $\underline{\Omega}^{g \cdot \omega}=\operatorname{Ad} \underline{\Omega}^{\omega}$, where Ad is the natural adjoint action of the gauge group on $E_{\mathfrak{g}}$. A computation readily shows that if $\omega$ is a Yang-Mills heat flow, then so is $g \cdot \omega$, albeit on a smaller subset of $M$ if $U \neq M$, and the norm of the curvature form $\underline{\Omega}^{\omega}$ (as well as its covariant derivatives) is invariant under the action of the gauge group.

When speaking of the convergence of a sequence $\left\{\omega_{i}\right\}$ of connections (with corresponding $E_{\mathfrak{g}}$-valued one-forms $\left\{u_{i}\right\}$ ), we shall say that $\left\{u_{i}\right\}$ (or $\left\{\omega_{i}\right\}$ ) converges locally uniformly on $U \subset M$ modulo gauge if there exists a sequence $\left\{g_{i}: U \rightarrow E_{G}\right\}$ of elements of the gauge group for which the $E_{\mathfrak{g}}$-valued one-form $\left\{\tilde{u}_{i}\right\}$ corresponding to $\left\{g_{i} \cdot \omega_{i}\right\}$ (and some fixed connection $\omega_{0}$ ) converges locally uniformly on $U \subset M$. Likewise, by convergence locally uniformly in $C^{\infty}$ we shall mean that $\left\{\widetilde{u}_{i}\right\}$ converges locally uniformly in $C^{\infty}$ on coördinate patches. For our purposes, working with the trivial connection with respect to some local section will be sufficient.

When working locally, the action of the gauge group is essentially equivalent to choosing a different representative local one-form of the connection; indeed, if $\sigma$ : $U \rightarrow P$ and $\sigma^{\prime}: U^{\prime} \rightarrow P$ are two local sections of $P$, then there exists a smooth mapping $g: U \cap U^{\prime} \rightarrow G$ such that $\sigma^{\prime} \cdot g=\sigma, \cdot$ being the right action of $G$ on $P$, and the associated local connection forms $A^{\sigma}$ and $A^{\sigma^{\prime}}$ are related by

$$
A_{i}^{\sigma^{\prime}}=\operatorname{Ad}_{g}\left(A_{i}^{\sigma}\right)-\partial_{i} g \cdot g^{-1}
$$

We shall take this view point for the sake of concreteness.

\subsubsection{Common Properties}

We recall some well-known facts common to the Yang-Mills and harmonic map heat flows; these results are essentially contained in [20] for the harmonic map heat flow and [41] for the Yang-Mills heat flow, albeit in a slightly different form. For the convenience of the reader, we sketch their proofs.

In both cases, local control on the fundamental form $\psi$ ensures local control on the derivatives of $\psi$ of all orders in the following sense:

Lemma 2.1 (Higher regularity) If the estimate $r^{2 k} \sup _{Q_{r}(X, T)} \frac{1}{2}|\psi|^{2} \leq c_{0}$ holds for some $c_{0}>0,0<r<$ inj $_{X}$ and $X \in M$, then for each $i \in \mathbb{N}$ there exists a constant $c_{i}>0$ depending only on $c_{0}$, bounds on the covariant derivatives of the Riemann curvature tensor in $B_{r}(X)$ up to order $i$ and the geometry of the target of the underlying flow such that 


$$
r^{2(k+i)} \sup _{Q_{r / 2}(X, T)} \frac{1}{2}\left|\nabla^{i} \psi\right|^{2} \leq c_{i} .
$$

A variant of this lemma was established in [20, Theorem 2.2] for the case of the harmonic map heat flow and [41, Theorem 2.2] for the case of the Yang-Mills heat flow. In the former case, the $\left\{c_{i}\right\}$ depends on bounds on the sectional curvature of $N$ and in the latter the structure constants of $\mathfrak{g}$. For completeness' sake, the proof of this version shall now be sketched.

We first recall the following localised weak maximum principle due to Ecker and Huisken [15]; its proof may be found in [13, Prop. 3.17] under the assumption that $M$ evolves by mean curvature flow, though the proof carries over mutatis mutandis to our setting.

Theorem 2.2 (Localised maximum principle) Fix $t_{1}, t_{2}>0$ and let $\phi: M \times\left[t_{1}, t_{2}[\rightarrow\right.$ $\left[0, \infty\left[\right.\right.$ be a $C^{2}$ function such that $\phi(\cdot, t) \in C_{0}^{2}(M)$ for all $t \in\left[t_{1}, t_{2}[\right.$ and

$$
\phi+|\nabla \phi|+\left|\partial_{t} \phi\right|+\left|\nabla^{2} \phi\right|+\frac{|\nabla \phi|^{2}}{\phi} \leq c_{\phi}
$$

for some constant $c_{\phi}>0$. Furthermore suppose that $f: M \times\left[t_{1}, t_{2}[\rightarrow[0, \infty[\right.$ is a $C^{2}$ function such that

$$
\left(\partial_{t}-\Delta\right) f \leq-a_{0} f^{2}-a_{1} \cdot f+\langle X, \nabla f\rangle+a_{2}
$$

on $U:=\{(x, t): \phi(x, t)>0\}$ with $a_{0}>0, a_{2} \in \mathbb{R}, a_{1}: U \rightarrow[0, \infty[$ and $X: U \rightarrow T M$ a time-dependent vector field such that $a_{3}:=\sup _{U} \frac{|X|}{\sqrt{1+a_{1}}}<\infty$. Then for all $t \in\left[t_{1}, t_{2}[\right.$, we have that

$$
\max _{M}(f \cdot \phi)(\cdot, t) \leq \max _{M}(f \cdot \phi)\left(\cdot, t_{1}\right)+\delta_{1},
$$

where

$$
\delta_{1}=\sqrt{\frac{4}{3}\left(\frac{\left(4+n+\frac{1}{4} a_{3}^{2}\right)^{2}}{a_{0}^{2}}+\frac{a_{2} c_{\phi}^{2}}{a_{0}}\right)} .
$$

Proof of Lemma 2.1 By rescaling the flow and crudely rescaling the metric, we may without loss of generality suppose that $r=1$ and $(x, t)=(0,0) \in \mathbb{R}^{n}$, since the scaling behaviour of $g, \psi$, the Riemann curvature tensor and its covariant derivatives would then establish the desired estimate. Set $f_{k}=\frac{1}{2}\left|\nabla^{k} \psi\right|^{2}$. By successively differentiating (2.3) in both cases, we see that whenever

$$
\sup _{Q} f_{i} \leq c_{i}
$$


for $i \in\{0, \ldots, k-1\},\left\{c_{i}\right\}_{i=0}^{k-1} \subset \mathbb{R}$ and $Q \subset Q_{1}(0,0)$ open, then

$$
\left(\partial_{t}-\Delta\right) f_{k} \leq-2 f_{k+1}+C_{k}\left(1+f_{k}\right)
$$

with $C_{k}$ depending (polynomially) on $\left\{c_{i}\right\}_{i=0}^{k-1}$ and bounds on the covariant derivatives of the Riemann curvature tensor up to order $k$, as well as the structure constants of $\mathfrak{g}$ in the case of the Yang-Mills heat flow, and bounds on the second fundamental form of $N$ up to order $k+1$ in the case of the harmonic map heat flow (cf. [20, Theorem 2.2] and [41, Theorem 2.2]). We shall employ a variant of the Shi trick (cf. [33, Lemma 4.2]) in order to apply Theorem 2.2. To this end, assuming the bounds (2.13), set $g_{k}=f_{k} \cdot\left(\alpha_{k}+f_{k-1}\right)$, with $\alpha_{k}>0$ to be determined. We compute, writing L for the heat operator $\partial_{t}-\Delta$, that

$$
\begin{aligned}
\mathrm{L} g_{k}=\mathrm{L} f_{k} & \cdot\left(\alpha_{k}+f_{k-1}\right)+f_{k} \cdot \mathrm{L} f_{k-1}-2\left\langle\nabla f_{k}, \nabla f_{k-1}\right\rangle \\
\leq(4 \varepsilon- & 2) f_{k+1}\left(\alpha_{k}+f_{k-1}\right)+C_{k}\left(\alpha_{k}+f_{k-1}\right)+C_{k} g_{k} \\
& \quad+C_{k-1} f_{k}\left(1+f_{k-1}\right)+\left(-2+\frac{2}{\varepsilon} \cdot \frac{f_{k-1}}{\alpha_{k}+f_{k-1}}\right) f_{k}^{2},
\end{aligned}
$$

for every $\varepsilon>0$, where we have used the evolution inequality (2.14) as well as the inequality

$$
\begin{aligned}
\left|\left\langle\nabla f_{k}, \nabla f_{k-1}\right\rangle\right| & \leq\left|\nabla^{k} \psi\right|^{2} \cdot\left|\nabla^{k+1} \psi\right| \cdot\left|\nabla^{k-1} \psi\right| \\
& \leq 2 \varepsilon\left(\alpha_{k}+f_{k-1}\right) f_{k+1}+\frac{2}{\varepsilon\left(\alpha_{k}+f_{k-1}\right)} f_{k}^{2} f_{k-1},
\end{aligned}
$$

where the former inequality follows from Kato's inequality and the latter Young's inequality. Setting $\varepsilon=\frac{1}{2}$ eliminates the first term, and since $x \mapsto \frac{x}{\alpha_{k}+x}$ is monotone increasing, (2.13) together with another application of Young's inequality in the form

$$
C_{k-1} f_{k}\left(1+f_{k-1}\right) \leq \frac{C_{k-1}^{2}}{2}\left(1+c_{k-1}\right)^{2}+\frac{1}{2} f_{k}^{2}
$$

yields the inequality

$$
\mathrm{L} g_{k} \leq\left(-\frac{3}{2}+4 \frac{c_{k-1}}{\alpha_{k}+c_{k-1}}\right) f_{k}^{2}+C_{k} g_{k}+\widetilde{c}_{k}
$$

with $\widetilde{c}_{k}=C_{k}\left(\alpha_{k}+c_{k-1}\right)+\frac{C_{k-1}^{2}}{2}\left(1+c_{k-1}\right)^{2}$. Choosing $\alpha_{k}=\frac{13}{3} c_{k-1}$ which then implies that $g_{k} \leq \frac{16}{3} c_{k-1} f_{k}$, we arrive at the evolution inequality

$$
\begin{aligned}
\mathrm{L} g_{k} & \leq-\frac{27}{1024 c_{k-1}^{2}} g_{k}^{2}+C_{k} g_{k}+\widetilde{c}_{k} \\
& \leq-\frac{27}{2048 c_{k-1}^{2}} g_{k}^{2}+\left(\widetilde{c}_{k}+\frac{512 c_{k-1}^{2} C_{k}^{2}}{27}\right)
\end{aligned}
$$


which is of the form (2.11), where in the last line we applied Young's inequality. Now, for each $k \in \mathbb{N} \cup\{0\}$, set $r_{k}=\frac{1}{2}+\left(\frac{1}{4}\right)^{k}$ and define $\varphi_{k}: \mathbb{R}^{n} \times\left[-r_{k-1}^{2}, 0[\rightarrow[0, \infty[\right.$ such that

$$
\varphi_{k}(x, t)=\left(t+r_{k-1}^{2}\right) \cdot \max \left\{0,\left(r_{k-1}^{2}-|x|^{2}\right)^{3}\right\} .
$$

Then $\varphi_{k}\left(\cdot,-r_{k-1}^{2}\right) \equiv 0$, for each $\left.t \in\right]-r_{k-1}^{2}, 0\left[\right.$, supp $\varphi_{k}(\cdot, t)=\overline{B_{r_{k-1}}(0)}, \varphi_{k}$ satisfies the inequality (2.10) with $c_{\varphi_{k}}$ depending on $k$ as well as the constants $\Lambda_{ \pm \infty}$ arising in the geometry bounds (2.2), and we have the inequality

$$
\left.\varphi_{k}\right|_{Q_{k}(0,0)} \geq\left(r_{k-1}^{2}-r_{k}^{2}\right)^{4}=: \gamma_{k}
$$

Now, suppose that $\sup _{Q_{1}(0,0)} f_{0} \leq c_{0}$. Applying Theorem 2.2 to $f=g_{1}$ and $\phi=\varphi_{1}$, we immediately obtain a bound $\beta_{1}>0$ depending on $n$ and the coëfficients appearing on the right-hand side of (2.15), viz. $c_{0}, C_{0}$ and $C_{1}$, the latter of which depend appropriately on the underlying geometry, such that

$$
\sup _{B_{1}(0)}\left(g_{1} \cdot \varphi_{1}\right)(\cdot, t) \leq \beta_{1}
$$

for each $t \in\left[-1,0\left[\right.\right.$. Using (2.16), we obtain that $\sup _{Q_{r_{1}}(0,0)} g_{1} \leq \frac{\beta_{1}}{\gamma_{1}}$. Proceeding by induction, we see that

$$
\sup _{Q_{r_{k}}(0,0)} g_{k} \leq \frac{\beta_{k}}{\gamma_{k}}
$$

for each $k \in \mathbb{N}$ with $\beta_{k}>0$ depending only on $n, c_{0}$ and the underlying geometry. Since $g_{k} \geq \alpha_{k} f_{k}$, we may proceed again by induction to obtain estimates

$$
\sup _{Q_{r_{k}}(0,0)} f_{k} \leq c_{k}
$$

for each $k \in \mathbb{N}$, where $c_{k}$ depends on $n, c_{0}$ and the underlying geometry as before. Since $\frac{1}{2}<r_{k} \leq 1$ for all $k$, this establishes the claim.

Remark 2.3 By carefully inspecting the expression (2.12), it may be readily checked in the process of the inductive argument that the $\left\{c_{i}\right\}_{i=1}^{\infty}$ in (2.9) may be bounded from above by an expression depending polynomially on $c_{0}$ and the underlying geometric bounds.

Since we are restricting our attention to the harmonic map heat flow with compact target manifold, we automatically have boundedness of $u$ and, by Lemma 2.1, once we have a bound on $\mathrm{d} u$ in a parabolic cylinder, we obtain local bounds on $\nabla^{l} \mathrm{~d} u$ for all $l \in \mathbb{N}$ in a slightly smaller parabolic cylinder. The situation is more complicated in the case of the Yang-Mills heat flow, where a bound on the curvature two-form does not equate to a bound on the differential of $u$ owing to gauge invariance. However, 
given a bound on the curvature two-form in a parabolic cylinder, we may by suitably choosing a local section (or equivalently by acting an element of the gauge group) deduce the boundedness of the local connection form and all of its derivatives in a slightly smaller parabolic cylinder, the key ingredient being Uhlenbeck's Coulomb gauge theorem [38].

Lemma 2.4 (Nice gauge lemma) If $r^{4} \sup _{Q_{r}(X, T)} \frac{1}{2}\left|\underline{\Omega}^{\omega}\right|^{2} \leq c_{0}$ for some $0<r<i_{0}$ and $c_{0}>0$, then there exist constants $\left.\left.\theta \in\right] 0, \frac{1}{4}\right]$ and $\left.\left\{\alpha_{i}\right\}_{i=0}^{\infty} \subset\right] 0, \infty[$ depending only on $c_{0}, n, i_{0}$, bounds on the covariant derivatives of the Riemann curvature tensor and the structure constants of $\mathfrak{g}$, and a local section $\sigma: B_{2 \theta r}(X) \rightarrow P$ such that for all $i \in \mathbb{N} \cup\{0\}$,

$$
r^{2(1+i)} \sup _{Q_{\theta r}(X, T)} \frac{1}{2}\left|\nabla^{i} \sigma^{*} \omega\right|^{2} \leq \alpha_{i} .
$$

Proof Let $t_{0}=T-\left(\frac{r}{2}\right)^{2}$ and write $g_{\delta}$ for the Euclidean metric on $B_{r}(X)$ defined in geodesic normal coördinates at $X$. Note that we have a bound of the form

$$
\left|g-g_{\delta}\right|_{g_{\delta}}\left(\vartheta_{X}(x)\right) \leq \alpha(n) \cdot \sup _{B_{i_{0}}(X)}|R|_{g_{\delta}} \cdot|x|^{2} \leq \beta|x|^{2}
$$

where $\vartheta_{X}: B_{\operatorname{inj}_{X}}(0) \subset \mathbb{R}^{n} \rightarrow B_{\operatorname{inj}_{X}}(X) \subset M$ is the associated geodesic normal parametrisation of $B_{\operatorname{inj}_{X}}(X), \alpha(n)$ is a constant depending only on $n, R$ is the Riemann curvature tensor of $g$ and $\beta$ is any upper bound for the coëfficient of $|x|^{2}$ in the middle expression of this inequality. Since $\int_{B_{\lambda r}(X)} \frac{1}{2}\left|\underline{\Omega}^{\omega}\right|^{\frac{n}{2}}\left(\cdot, t_{0}\right) \stackrel{\lambda \searrow 0}{\longrightarrow} 0$, it follows from Uhlenbeck's Coulomb gauge theorem that there exists a $\left.\theta \in] 0, \frac{1}{4}\right]$ depending only on $c_{0}, n, \beta$ and the structure constants of $\mathfrak{g}$, and a local section $\sigma: B_{2 \theta r}(X) \rightarrow P$ such that the Coulomb gauge condition $\sum_{i=1}^{n} \partial_{i}\left(\sigma^{*} \omega\left(\cdot, t_{0}\right), \partial_{i}\right)=0$ holds as well as a scale-invariant bound on the $W^{1, p}$ norm of $\sigma^{*} \omega\left(\cdot, t_{0}\right)$ on $B_{2 \theta r}(X)$ in terms of the $L^{p}$ norm of $\underline{\Omega}^{\omega}$ for all $p \geq \frac{n}{2}$. Choosing $\theta$ smaller depending on $\beta$ and $n$ if necessary, the Coulomb gauge condition together with (2.8) implies that $\sigma^{*} \omega\left(\cdot, t_{0}\right)$ solves an elliptic system so that by standard techniques (cf. [24, Sect. 3.5] and [12, Sect. 4]), scale-invariant estimates

$$
r^{2(1+i)} \sup _{B_{\theta r}(X)} \frac{1}{2}\left|\nabla^{i} \sigma^{*} \omega\left(\cdot, t_{0}\right)\right|^{2} \leq \beta_{i}
$$

follow, where the $\left\{\beta_{i}\right\}_{i=0}^{\infty}$ depend only on $c_{0}$, bounds on the covariant derivatives of $R$ in $B_{i_{0}}(X)$ and the structure constants of $\mathfrak{g}$. Now, it follows from the bound on $\underline{\Omega}^{\omega}$ and Lemma 2.1 that on $Q_{\frac{r}{2}}(X, T)$,

$$
\partial_{t} \frac{1}{2}\left|\sigma^{*} \omega\right|^{2} \leq \frac{2 n \sqrt{c_{1}}}{r^{3}} \cdot \sqrt{\frac{1}{2}\left|\sigma^{*} \omega\right|^{2}}
$$


An integration and application of (2.18) then yield the inequality

$$
r^{2} \sup _{B_{\theta r}(X)} \frac{1}{2}\left|\sigma^{*} \omega(\cdot, t)\right|^{2} \leq \alpha_{0}:=\left(\beta_{0}+n \sqrt{c_{1}}\right)^{2}
$$

for all $t \in\left[t_{0}, T\right.$, which implies (2.17) in the case $i=0$. The remaining estimates follow similarly by induction.

Remark 2.5 We may leverage Lemma 2.4 to obtain bounds on the covariant derivatives of the section $u$ associated with the Yang-Mills heat flow as follows: Let $\varphi: M \rightarrow$ $\left[0, \infty\right.$ [ be any smooth function such that $\left.\varphi\right|_{B_{\theta r}(X)} \equiv 1$ and supp $\varphi \Subset B_{2 \theta r}(0)$. Setting $\omega_{0}=\varphi \circ \pi \cdot \omega_{\sigma}+(1-\varphi \circ \pi) \omega_{1}$, where $\pi: P \rightarrow M$ is the projection map of the principal bundle, $\omega_{\sigma}$ is the trivial connection associated with the local section $\sigma$ and $\omega_{1}$ is any other smooth connection on $P$, we have the equality

$$
u(x, t)=\Psi_{\sigma}\left(x, \sigma^{*} \omega(x, t)\right)
$$

for $(x, t) \in Q_{\theta r}(X, T)$, where $\Psi_{\sigma}: B_{2 \theta r}(X) \times \mathfrak{g} \rightarrow E_{\mathfrak{g}}$ is the local bundle parametrisation of $E_{\mathfrak{g}}$ associated with $\sigma$ acting on the former part of the tensor product $\mathfrak{g} \otimes T_{x}^{*} M$. This immediately implies that

$$
r^{2(1+i)} \sup _{Q_{\theta r}(X, T)} \frac{1}{2}\left|\nabla^{i} u\right|^{2} \leq \alpha_{i}
$$

under the hypotheses of the lemma. Moreover, in the light of these bounds, we obtain similar estimates with the trivial connection with respect to $\sigma$ in place of $\nabla$.

\subsection{Heat Balls and Local Monotonicity}

We now turn our attention to the monotonicity properties of the harmonic map and Yang-Mills heat flows.

For fixed $(x, t) \in \Omega \times] 0, T], r>0$ and $k<\frac{n}{2}$, the generalised Euclidean $(n-2 k)$ heat ball of radius $r$ centred at $(x, t)$ is defined by

$$
\begin{aligned}
E_{r}^{n-2 k}(x, t) & \left.=\{(y, s) \in \Omega \times] 0, T]: \frac{1}{(4 \pi(t-s))^{\frac{n-2 k}{2}}} \exp \left(\frac{\mathfrak{r}(y, x)^{2}}{4(s-t)}\right)>\frac{1}{r^{n-2 k}}\right\} \\
& =\bigcup_{\left.s \in] t-\frac{r^{2}}{4 \pi}, t[\cap] 0, T\right]}\left(B_{R_{r}^{n-2 k}(s-t)}(x) \cap \Omega\right) \times\{s\},
\end{aligned}
$$

where $R_{r}^{n-2 k}(\sigma)=\sqrt{2(n-2 k) \sigma \log \left(-\frac{4 \pi \sigma}{r^{2}}\right)}$ for $\left.\sigma \in\right]-\frac{r^{2}}{4 \pi}, 0[$. It may be seen from the definition of $E_{r}^{n-2 k}(x, t)$ that it is relatively compact in $M \times[0, T]$. Moreover, we have the inequality $R_{r}^{n-2 k}(\sigma) \leq \sqrt{\frac{n-2 k}{2 \pi e}} r$ so that setting $c_{n, k}:=\max \left\{\frac{1}{\sqrt{4 \pi}}, \sqrt{\frac{n-2 k}{2 \pi e}}\right\}$, 
we have the inclusion

$$
E_{r}^{n-2 k}(x, t) \subset Q_{c_{n, k} r}(x, t)
$$

For technical reasons, we shall restrict our attention to small $r$ so that $E_{r}^{n-2 k}(x, t) \subset$ $\Omega \times] 0, T[$.

We now state the local monotonicity principle for these flows. For simplicity, we introduce the shorthand notation

$$
e_{k}[u, g]_{(x, t)}=\frac{1}{2}|\psi|^{2} \cdot\left(\partial_{t} \phi_{(x, t)}+\left|\nabla \phi_{(x, t)}\right|^{2}\right)-\left\langle\nabla \phi _ { ( x , t ) } \left\llcorner\psi, \partial_{t} u+\nabla \phi_{(x, t)}\llcorner\psi\rangle,\right.\right.
$$

where $\phi_{(x, t)}(y, s)=\frac{\mathfrak{r}(x, y)^{2}}{4(s-t)}-\frac{n-2 k}{2} \log (4 \pi(t-s))$. Note that the right-hand side implicitly depends on the choice of metric $g$. The following was established by Ecker [14] in the case of the harmonic map heat flow with $M$ Euclidean and more generally for the Yang-Mills and harmonic map heat flows on Riemannian manifolds by the author [1,3].

Theorem 2.6 (Local monotonicity) Fix $x \in \Omega$ and suppose $u$ is a harmonic map or Yang-Mills heat flow on $Q_{R}(x, t) \subset \Omega \times \mathbb{R}$ for $R \leq i_{0}$. Then there exist $r_{0}>0$ and $\xi_{k} \in C^{\infty}(] 0, \infty[)$ with $\lim _{s \searrow 0} \xi_{k}(s)=0$ depending on the geometry of $\Omega$ such that the heat ball energy

$$
r \mapsto \frac{1}{r^{n-2 k}} \iint_{E_{r}^{n-2 k}(x, t)} e^{\xi_{k}(t-s)} e_{k}[u, g]_{(x, t)}(y, s) d v o l_{g}(y) d s
$$

is monotone nondecreasing for $r<r_{0}$ whenever the integrand is defined and summable over $E_{r_{0}}^{n-2 k}(x, t)$. If $(M, g)=\left(\mathbb{R}^{n}, g_{\delta}\right)$, then $\xi_{k} \equiv 0$ and (2.19) is constant iff $u$ is self-similar about $(x, t)$ in $E_{r_{0}}^{n-2 k}(x, t)$ (modulo gauge).

Remark 2.7 The geometric quantities $r_{0}$ and $\xi_{k}$ are explicitly given by the following expressions:

$$
\begin{aligned}
r_{0} & =\frac{R}{2 c_{n, k}} ; \\
\xi_{k}(\sigma) & =\sigma\left[\left((n-1) \Lambda_{\infty}^{+}-2 k \Lambda_{-\infty}^{-}\right) \log \left(\frac{e}{4 \pi \sigma}\right)^{\frac{n-2 k}{2}}\right],
\end{aligned}
$$

where $\Lambda_{ \pm}$arise from the geometry bounds (2.2).

Remark 2.8 (Scale invariance) We may more explicitly write $e_{k}[u, g]_{(x, t)}(y, s)$ in geodesic normal coördinates about $(x, t)$ as

$$
\frac{1}{2}|\psi|^{2}(y, s) \cdot \frac{n-2 k}{2(t-s)}-\left\langle\frac { y } { 2 ( s - t ) } \left\llcorner\psi(y, s), \partial_{t} u+\frac{y}{2(s-t)}\llcorner\psi(y, s)\rangle .\right.\right.
$$


In particular, using this local expression, we have that

$$
\begin{aligned}
& \frac{1}{r^{n-2 k}} \iint_{E_{r}^{n-2 k}(x, t)} e^{\xi_{k}(t-s)} e_{k}[u, g]_{(x, t)}(y, s) \operatorname{dvol}_{g}(y) \mathrm{d} s \\
& \quad=\frac{1}{(r / \lambda)^{n-2 k}} \iint_{E_{r / \lambda}^{n-2 k}(0,0)} e^{\xi_{k}\left(-\lambda^{2} s\right)} e_{k}\left[u_{\lambda}^{(x, t)}, g_{\lambda}^{x}\right]_{(0,0)}(y, s) \cdot \sqrt{g_{\lambda}^{x}}(y) \mathrm{d} y \mathrm{~d} s
\end{aligned}
$$

for any $\lambda>0$. We shall use this (approximate) scale invariance property in the sequel.

Remark 2.9 (Nonnegativity) By monotonicity and scale invariance, if $u$ is smooth on $\overline{Q_{R}(x, t)}$, then for all $r<r_{0}$,

$$
\begin{aligned}
& \frac{1}{r^{n-2 k}} \iint_{E_{r}^{n-2 k}(x, t)} e^{\xi_{k}(t-s)} e_{k}[u, g]_{(x, t)}(y, s) \operatorname{dvol}_{g}(y) \mathrm{d} s \\
& \quad \geq \lim _{\lambda \searrow 0} \frac{1}{\lambda^{n-2 k}} \iint_{E_{\lambda}^{n-2 k}(x, t)} e^{\xi_{k}(t-s)} e_{k}[u, g]_{(x, t)}(y, s) \operatorname{dvol}_{g}(y) \mathrm{d} s \\
& \quad=\lim _{\lambda \searrow 0} \iint_{E_{1}^{n-2 k}(0,0)} e^{\xi_{k}\left(-\lambda^{2} s\right)} e_{k}\left[u_{\lambda}^{(x, t)}, g_{\lambda}^{x}\right]_{(0,0)}(y, s) \cdot \sqrt{g_{\lambda}^{x}}(y) \mathrm{d} y \mathrm{~d} s=0,
\end{aligned}
$$

where the last equality follows from the dominated convergence theorem. Therefore, the quantity (2.19) is nonnegative in the case where $u$ is smooth in a neighbourhood of $x$ up to and including time $t$. In the special case where $(M, g)=\left(\mathbb{R}^{n}, g_{\delta}\right)$ and $u$ is self-similar about $(x, t)$, the heat ball integrand takes the form

$$
e_{k}\left[u, g_{\delta}\right]_{(x, t)}(y, s)=\frac{1}{2}|\psi|^{2}(y, s) \cdot \frac{n-2 k}{2(t-s)},
$$

which is nonnegative on $E_{r}^{n-2 k}(x, t)$ regardless of whether $u$ is smooth up to and including time $t$.

It was shown in [3] that the quantity (2.19) is finite for $r \leq r_{0}$ whenever $\frac{1}{2}|\psi|^{2}$ is summable over a suitable parabolic cylinder containing $E_{r_{0}}^{n-2 k}(x, t)$. In fact, we have the following estimate.

Lemma 2.10 ( $L^{2}$ estimate) Suppose $u$ is a harmonic map or Yang-Mills heat flow on $\Omega \times[0, T[$. Then the estimate

$$
\frac{1}{r^{n-2 k}} \iint_{E_{r}^{n-2 k}(x, t)}\left|e_{k}[u, g]_{(x, t)}\right|(y, s) e^{\xi_{k}(t-s)} d \operatorname{vol}_{g}(y) d s \leq \frac{\gamma_{n, k}}{r^{n-2 k+2}} \iint_{Q_{4 c_{n, k}}(x, t)} \frac{1}{2}|\psi|^{2}
$$

holds whenever $\left.Q_{4 c_{n, k} r}(x, t) \subset \Omega \times\right] 0, T\left[\right.$ and $r \leq \frac{r_{0}}{2}$, where $\gamma_{n, k}$ depends on $n, k$ and the geometry of $\Omega$. 
Proof In [3, Remark 5.8], it was shown that

$$
\begin{aligned}
& \frac{1}{r^{n-2 k}} \iint_{E_{r}^{n-2 k}(x, t)}\left|e_{k}[u, g]_{(x, t)}\right|(y, s) \operatorname{dvol}_{g}(y) \mathrm{d} s \\
& \quad \leq c_{1}\left(\frac{1}{r^{n-2 k+2}} \iint_{Q_{2 c_{n, k}}(x, t)} \frac{1}{2}|\psi|^{2}+\frac{1}{r^{n-2 k}} \int_{B_{2 c_{n, k} r_{0}(x)}} \frac{1}{2}|\psi|^{2}\left(\cdot, t-\frac{r^{2}}{4 \pi}\right)\right),
\end{aligned}
$$

where $c_{1}$ depends on $n, k$ and the geometry of $\Omega$. Now, it may be shown using the methods of [2] that

$$
\begin{aligned}
& \frac{\mathrm{d}}{\mathrm{d} t} \int_{M} \frac{1}{2}|\psi|^{2} \varphi(\cdot, t) \\
& \quad=-\int_{M}\left|\partial_{t} u\right|^{2} \varphi+\int_{M} \frac{1}{2}|\psi|^{2}\left(\partial_{t}-\Delta\right) \varphi+\left\langle\nabla^{2} \varphi, \sum_{i, j}\left\langle\partial_{i}\left\llcorner\psi, \partial_{j}\llcorner\psi\rangle \mathrm{d} x^{i} \otimes \mathrm{d} x^{j}\right\rangle\right.\right.
\end{aligned}
$$

for a smooth function $\varphi: M \times[0, T[\rightarrow[0, \infty[$ such that $\varphi(\cdot, t)$ is compactly supported in $M$ for each $t$. We fix a smooth function $\chi: \mathbb{R} \rightarrow[0,1]$ such that $\chi(t)=1$ for $t<\frac{1}{2}$ and $\chi(t)=0$ for $t>1$. Setting $\varphi(y, s)=\chi\left(\left(\frac{\mathfrak{r}(y, x)}{4 c_{n, k} r}\right)^{2}+\frac{t-s}{\left(4 c_{n, k} r\right)^{2}}\right)$, it follows that $\operatorname{supp} \varphi(\cdot, s) \subset B_{4 c_{n, k} r}(x), \varphi(\cdot, s)=0$ for $s \leq t-\left(4 c_{n, k} r\right)^{2}$, and $\varphi \equiv 1$ on $Q_{2 c_{n, k} r}(x, t)$. Moreover, by the Hessian estimate (2.2), (2.22) implies that

$$
\frac{\mathrm{d}}{\mathrm{d} t} \int_{M} \frac{1}{2}|\psi|^{2} \varphi(\cdot, t) \leq \frac{c_{2}}{r^{2}} \int_{B_{4 c_{n, k^{r}}(x)}} \frac{1}{2}|\psi|^{2}(\cdot, t)
$$

where $c_{2}$ depends on $n, k$ and the geometry of $\Omega$. Integrating from $t-\left(4 c_{n, k} r\right)^{2}$ to $t-\frac{r^{2}}{4 \pi}$, we obtain

$$
\int_{B_{2 c_{n, k} r}(x)} \frac{1}{2}|\psi|^{2}\left(\cdot, t-\frac{r^{2}}{4 \pi}\right) \leq \int_{M} \frac{1}{2}|\psi|^{2} \varphi\left(\cdot, t-\frac{r^{2}}{4 \pi}\right) \leq \frac{c_{2}}{r^{2}} \iint_{Q_{4 c_{n, k} r}(x, t)} \frac{1}{2}|\psi|^{2},
$$

where we have used the fact that $t-\frac{r^{2}}{4 \pi}>t-\left(2 c_{n, k} r\right)^{2}$. Substituting this into (2.21) and using the boundedness of $\xi_{k}$ on bounded subsets of ]0, $\infty$ [ then yields (2.20).

Remark 2.11 (Local energy estimate) Using (2.22) with

$$
\varphi(y, s)=\chi\left(\left(\frac{\mathfrak{r}(y, x)}{2 r}\right)^{2}+\frac{t-s}{(2 r)^{2}}\right)
$$


and $x \in \Omega$, we may similarly establish the local energy bound

$$
\int_{B_{r}(x)} \frac{1}{2}|\psi|^{2}(\cdot, s) \leq \frac{c}{r^{2}} \int_{Q_{2 r}(x, t)} \frac{1}{2}|\psi|^{2}
$$

for $r \leq \frac{i_{0}}{2}$ with $\psi$ arising from a harmonic map or Yang-Mills heat flow on $Q_{2 r}(x, t) \subset$ $\Omega \times] 0, T[, s \in] t-r^{2}, t$ [ and $c>0$ depending only on the geometry of $\Omega$, which leads to the bound

$$
\int_{K} \frac{1}{2}|\psi|^{2}(\cdot, t) \leq \widetilde{c} \cdot \iint_{\Omega \times] 0, T[} \frac{1}{2}|\psi|^{2}
$$

for $t \in] T-\min \left\{\frac{i_{0}^{2}}{4}, \frac{1}{2} \operatorname{dist}(K, \partial \Omega)\right\}^{2}, T[$ and $K \Subset \Omega$, where $\widetilde{c}$ depends on $\operatorname{dist}(K, \partial \Omega)$ and the geometry of $\Omega$.

\section{The Local Regularity Theorem}

We now turn our attention to the promised local regularity theorem. Let

$$
I_{k}(u, g ; x, t, r):=\frac{1}{r^{n-2 k}} \iint_{E_{r}^{n-2 k}(x, t)} e^{\xi_{k}(t-s)} e_{k}[u, g]_{(x, t)}(y, s) \operatorname{dvol}_{g}(y) \mathrm{d} s
$$

be the heat ball energy of Theorem 2.6.

Theorem 3.1 (Local regularity) There exist geometric constants $\varepsilon, C>0$ such that for any $R \leq i_{0}$ and Yang-Mills or harmonic map heat flow $u$ on $Q_{R}(X, T) \subset \Omega \times \mathbb{R}$, the implication

$$
\sup _{(x, t) \in Q_{R / 2}(X, T)} I_{k}\left(u, g ; x, t, \frac{R}{2 c_{n, k}}\right)<\varepsilon \Rightarrow R^{2 k} \sup _{Q_{R / 4}(X, T)} \frac{1}{2}|\psi|^{2} \leq C
$$

holds.

We first establish the following lemma whose proof relies on a blow-up argument akin to that of [42] and [13, Theorem 5.6].

Lemma 3.2 There exist geometric constants $\varepsilon, C^{\prime}>0$ such that for any $R \leq i_{0}$ and Yang-Mills or harmonic map heat flow u on $\overline{Q_{R}(X, T)} \subset \Omega \times \mathbb{R}$, the implication

$\sup _{(x, t) \in Q_{R / 2}(X, T)} I_{k}\left(u, g ; x, t, \frac{R}{2 c_{n, k}}\right)<\varepsilon \Rightarrow \sup _{\alpha \in] 0,1[}\left(\frac{\alpha R}{2}\right)^{2 k} \sup _{Q_{(1-\alpha) R / 2}(X, T)} \frac{1}{2}|\psi|^{2} \leq C^{\prime}$

holds.

Proof We proceed by contradiction. Suppose that this result is false. Then there exist a sequence $\left.\left.\left\{R_{j}\right\}_{j \in \mathbb{N}} \subset\right] 0, i_{0}\right]$ and a sequence of Yang-Mills or harmonic map heat flows 
$\left\{u_{j}\right\}_{j \in \mathbb{N}}$ with associated fundamental forms $\left\{\psi_{j}\right\}_{j \in \mathbb{N}}$, each defined on $\overline{Q_{R_{j}}\left(X_{j}, T_{j}\right)} \subset$ $\Omega \times \mathbb{R}$ for each $j \in \mathbb{N}$, such that the $\varepsilon$-regularity condition, i.e. the antecedent of the implication in the theorem, holds with $\varepsilon=\frac{1}{j}, R=R_{j}, u=u_{j}$ and $\psi=\psi_{j}$, but

$$
\left(\beta_{j}\right)^{2 k}:=\sup _{\alpha \in] 0,1[}\left(\frac{\alpha R_{j}}{2}\right)^{2 k} \sup _{Q_{(1-\alpha) \frac{R_{j}}{2}}(X, T)} \frac{1}{2}\left|\psi_{j}\right|^{2} \stackrel{j \rightarrow \infty}{\longrightarrow} \infty .
$$

By smoothness, there exist $\left.\left.\alpha_{j} \in\right] 0,1\right]$ and $\left(x_{j}, t_{j}\right) \in \overline{Q_{\left(1-\alpha_{j}\right)} \frac{R_{j}}{2}}\left(X_{j}, T_{j}\right)$ such that

$$
\left(\beta_{j}\right)^{2 k}=\left(\frac{\alpha_{j} R_{j}}{2}\right)^{2 k} \cdot \frac{1}{2}\left|\psi_{j}\right|^{2}\left(x_{j}, t_{j}\right) .
$$

By passing to a subsequence if necessary, we may assume that $\left\{x_{j}\right\}_{j \in \mathbb{N}} \subset \bar{\Omega}$ is a convergent sequence. Now, note that:

(1) There holds

$$
\begin{aligned}
& \sup _{Q_{\left(1-\frac{\alpha_{j}}{2}\right) \frac{R_{j}}{2}}(X, T)} \frac{1}{2}\left|\psi_{j}\right|^{2}=\left(\frac{4}{\alpha_{j} R_{j}}\right)^{2 k} \cdot\left(\left(\frac{\alpha_{j} R_{j}}{4}\right)^{2 k} \sup _{Q_{\left(1-\frac{\alpha_{j}}{2}\right) \frac{R_{j}}{2}}(X, T)} \frac{1}{2}\left|\psi_{j}\right|^{2}\right) \\
& \leq\left(\frac{4 \beta_{j}}{\alpha_{j} R_{j}}\right)^{2 k} \text {. }
\end{aligned}
$$

(2) We have the inclusion $Q_{\frac{\alpha_{j}}{2} \cdot \frac{R_{j}}{2}}\left(x_{j}, t_{j}\right) \subset Q_{\left(1-\frac{\alpha_{j}}{2}\right) \frac{R_{j}}{2}}(X, T)$.

Altogether, we have

$$
\sup _{\frac{Q_{\frac{\alpha_{j} R_{j}}{4}}\left(x_{j}, t_{j}\right)}{2}} \frac{1}{2}\left|\psi_{j}\right|^{2} \leq\left(\frac{4 \beta_{j}}{\alpha_{j} R_{j}}\right)^{2 k}=: \lambda_{j}^{-2 k} .
$$

We now rescale $u_{j}$ appropriately about $\left(x_{j}, t_{j}\right)$. For each $j \in \mathbb{N}$, set

$$
u_{j}^{\prime}(x, t)=\left(u_{j}\right)_{\lambda_{j}}^{\left(x_{j}, t_{j}\right)} .
$$

This defines a smooth harmonic map (resp. Yang-Mills) heat flow

$$
u_{j}^{\prime}: \overline{Q_{\beta_{j}}(0,0)} \rightarrow V \otimes \Lambda^{k-1} T^{*} \mathbb{R}^{n}
$$

with respect to the metric $g_{\lambda_{j}}^{x_{j}}$. Moreover, writing $\psi_{j}^{\prime}$ for the fundamental form associated with $u_{j}^{\prime}$, (3.2) implies that

$$
\frac{1}{2}\left|\psi_{j}^{\prime}\right|^{2}(0,0)=\lambda_{j}^{2 k} \cdot \frac{1}{2}\left|\psi_{j}^{\prime}\right|^{2}\left(x_{j}, t_{j}\right)=\left(\frac{1}{2}\right)^{2 k}
$$


and, in the light of (3.3), we have $\sup _{\overline{Q_{\beta_{j}}(0,0)}} \frac{1}{2}\left|\psi_{j}^{\prime}\right|^{2} \leq 1$. Since $\beta_{j} \geq 1$ for sufficiently large $j$, we also have that $\left.\sup _{\overline{Q_{1}(0,0)}}\left|\frac{1}{2}\right| \psi_{j}^{\prime}\right|^{2} \leq 1$ for sufficiently large $j$ and therefore, by Lemma 2.1, we have that

$$
\frac{\sup }{Q_{\frac{1}{2}}(0,0)} \frac{1}{2}\left|\nabla^{m} \psi_{j}^{\prime}\right|^{2} \leq c_{m}
$$

for all $m \in \mathbb{N}$, where $c_{m}$ depends only on $n$, bounds on the covariant derivatives of the Riemann curvature tensor of $g$ up to order $m$ in $\Omega$, the structure constants of $\mathfrak{g}$ in the case of the Yang-Mills heat flow and bounds on the covariant derivatives of the second fundamental form of the target manifold up to order $m+1$ in the case of the harmonic map heat flow. Using Lemma 2.4 in the case of the Yang-Mills heat flow and the compactness of the target manifold in the case of the harmonic map heat flow, we furthermore obtain bounds of the form

$$
\frac{\sup }{Q_{\theta}(0,0)} \frac{1}{2}\left|\nabla^{m} u_{j}^{\prime}\right|^{2} \leq \widetilde{c}_{m}
$$

for all $m \in \mathbb{N} \cup\{0\}$, where $\widetilde{c}_{m}$ likewise depends on the geometry of $\Omega$ and either the structure constants of $\mathfrak{g}$ or the second fundamental form of $N$. Thus, by the ArzelàAscoli theorem, there exists a subsequence of $\left\{u_{j}^{\prime}\right\}$, which we again denote by $\left\{u_{j}^{\prime}\right\}$, and a smooth Yang-Mills or harmonic map heat flow $u_{\infty}: \overline{Q_{\theta}(0,0)} \rightarrow V \otimes \Lambda^{k-1} T^{*} \mathbb{R}^{n}$ with respect to the Euclidean metric $g_{\delta}$ such that $u_{j}^{\prime} \stackrel{j \rightarrow \infty}{\longrightarrow} u_{\infty}$ uniformly in $C^{\infty}$. From (3.4), we see that we must also have

$$
\frac{1}{2}\left|\psi_{\infty}\right|^{2}(0,0)=\left(\frac{1}{2}\right)^{2 k}
$$

Now, for $(x, t)=\left(x_{j}, t_{j}\right)$, the $\varepsilon$-regularity condition reads

$$
\frac{1}{\left(\frac{R_{j}}{2 c_{n, k}}\right)^{n-2 k}} \iint_{E_{\frac{R_{j}}{2 c_{n, k}}}^{n-2 k}\left(x_{j}, t_{j}\right)} e_{k}\left[u_{j}, g\right]_{\left(x_{j}, t_{j}\right)}(x, t) \cdot e^{\xi\left(t-t_{j}\right)} \operatorname{dvol}_{g}(x) \mathrm{d} t<\frac{1}{j} .
$$

For fixed $\bar{R}>0$ and sufficiently large $j$, we have that $\frac{\bar{R} \alpha_{j} c_{n, k}}{2 \beta_{j}}<1 \Leftrightarrow \lambda_{j} \bar{R}<\frac{R_{j}}{2 c_{n, k}}$ so that by monotonicity (Theorem 2.6), we also have

$$
\frac{1}{\left(\lambda_{j} \bar{R}\right)^{n-2 k}} \iint_{E_{\lambda_{j} R}^{n-2 k}\left(x_{j}, t_{j}\right)} e_{k}\left[u_{j}, g\right]_{\left(x_{j}, t_{j}\right)}(x, t) \cdot e^{\xi\left(t-t_{j}\right)} \operatorname{dvol}_{g}(x) \mathrm{d} t<\frac{1}{j} .
$$

Using scale invariance and nonnegativity (Remarks 2.8 and 2.9), we therefore have

$$
0 \leq \frac{1}{\bar{R}^{n-2 k}} \iint_{E_{\bar{R}}^{n-2 k}(0,0)} e_{k}\left[u_{j}^{\prime}, g_{\lambda_{j}}^{x_{j}}\right]_{(0,0)}(x, t) \cdot e^{\xi\left(-\lambda_{j}^{2} t\right)} \sqrt{g_{\lambda_{j}}^{x_{j}}(x)} \mathrm{d} x \mathrm{~d} t<\frac{1}{j}
$$


in geodesic normal coördinates about $x_{j}$. Choosing $\bar{R}$ so that $E_{\bar{R}}^{n-2 k}(0,0) \subset Q_{\theta}(0,0)$ and taking the limit $j \rightarrow \infty$ in (3.6), we obtain that

$$
\frac{1}{\bar{R}^{n-2 k}} \iint_{E_{\bar{R}}^{n-2 k}(0,0)} e_{k}\left[u_{\infty}, g_{\delta}\right]_{(0,0)} \equiv 0,
$$

i.e. $u_{\infty}$ is a smooth self-similar flow on $E_{\bar{R}}^{n-2 k}(0,0)$ by Theorem 2.6, but then selfsimilarity implies that

$$
\frac{1}{2}\left|\psi_{\infty}\right|^{2}(x, t)=r^{2 k} \cdot \frac{1}{2}\left|\psi_{\infty}\right|^{2}\left(r x, r^{2} t\right)
$$

for all $(x, t) \in E_{\bar{R}}^{n-2 k}(0,0)$ and $\left.r \in\right] 0,1\left[\right.$. Since $u_{\infty}$ is smooth on $\overline{E_{\bar{R}}^{n-2 k}(0,0)} \subset$ $\overline{Q_{\theta}(0,0)}$, taking the limit $r \searrow 0$ yields $\frac{1}{2}\left|\psi_{\infty}\right|^{2}(x, t)=0$ for $(x, t) \in E_{\bar{R}}^{n-2 k}(0,0)$. Taking the limit $(x, t) \rightarrow(0,0)$ then yields the equality $\frac{1}{2}\left|\psi_{\infty}\right|^{2}(0,0)=0$, which contradicts (3.5).

Proof of Theorem 3.1 Let $\varepsilon$ and $C^{\prime}$ be as in Lemma 3.2 and suppose $u: Q_{R}(X, T) \rightarrow$ $E \otimes \Lambda^{k-1} T^{*} M$ is a harmonic map or Yang-Mills heat flow satisfying the $\varepsilon$-regularity condition

$$
\sup _{(x, t) \in Q_{R / 2}(X, T)} I_{k}\left(u, g ; x, t, \frac{R}{2 c_{n, k}}\right)<\varepsilon .
$$

Consider $u_{\delta}: \overline{Q_{\frac{15}{16} R}(X, T)} \rightarrow E \otimes \Lambda^{k-1} T^{*} M$ defined by $u_{\delta}(y, s)=u(y, s-\delta)$ for $\delta \in] 0,\left(1-\left(\frac{15}{16}\right)^{2}\right) \frac{R^{2}}{4}\left[. u_{\delta}\right.$ again defines a harmonic map or Yang-Mills heat flow, and using Remark 2.8 as well as Theorem 2.6, we see that

$$
\begin{gathered}
\sup _{(x, t) \in Q_{\left(\frac{15}{16} R\right) / 2}(X, T)} I_{k}\left(u_{\delta}, g ; x, t, \frac{15 R / 16}{2 c_{n, k}}\right) \\
=\sup _{(x, t) \in Q_{\left(\frac{15}{16} R\right) / 2}(X, T-\delta)} I_{k}\left(u, g ; x, t, \frac{15 R / 16}{2 c_{n, k}}\right) \\
\leq \sup _{(x, t) \in Q_{R / 2}(X, T)} I_{k}\left(u, g ; x, t, \frac{R}{2 c_{n, k}}\right)<\varepsilon .
\end{gathered}
$$

so that Lemma 3.2 applies to $u_{\delta}$, i.e. for all $\left.\alpha \in\right] 0,1\left[\right.$ and $(x, t) \in Q_{(1-\alpha)\left(\frac{15}{16} R\right) / 2}(X, T)$, we have that

$$
\left(\frac{15}{32} \alpha R\right)^{2 k} \cdot \frac{1}{2}|\psi|^{2}(x, t-\delta) \leq C^{\prime} .
$$

Choosing $\alpha=\frac{7}{15}$ and taking the limit $\delta \searrow 0$ then yields the desired estimate with $C=\left(\frac{32}{7}\right)^{2 k} C^{\prime}$. 
Remark 3.3 (Extensibility up to $t=T$ ) The significance of the $\varepsilon$-regularity condition

$$
\sup _{(x, t) \in Q_{R / 2}(X, T)} I_{k}\left(u, g ; x, t, \frac{R}{2 c_{n, k}}\right)<\varepsilon
$$

is the following: If (3.7) holds for a harmonic map or Yang-Mills heat flow $u$ on $\Omega \times[0, T$ [ for some $R>0$, then Theorem 3.1 implies a scale-invariant bound on $|\psi|^{2}$ in $Q_{\frac{R}{4}}(X, T)$ so that after applying Lemma 2.1 and appealing to (2.4) and (2.8), we conclude that $\lim _{t \nearrow T} u(\cdot, t)$ exists uniformly in $C^{\infty}$ on $\overline{B_{\frac{R}{8}}(X)}$, i.e. $\left.u\right|_{Q_{\frac{R}{8}}(X, T)}$ is smoothly extensible to all of $\overline{Q_{\frac{R}{8}(X, T)}}$. Conversely, if $u$ is a smooth harmonic map or Yang-Mills heat flow on $\overline{Q_{R}(X, T)}$ for some $\left.R \in\right] 0, i_{0}$, then by Lemma 2.10 and Remark 2.9, we have for all $\lambda \leq \frac{1}{5} R$ that

$$
\begin{aligned}
0 & \leq \sup _{(x, t) \in Q_{\lambda / 2}(X, T)} I_{k}\left(u, g ; x, t, \frac{\lambda}{2 c_{n, k}}\right) \\
& \leq \frac{\gamma_{n, k} \cdot\left(2 c_{n, k}\right)^{n-2 k+2}}{\lambda^{n-2 k+2}} \iint_{Q_{\frac{5 \lambda}{2}}(X, T)} \frac{1}{2}|\psi|^{2} \\
& =\gamma_{n, k} \cdot\left(2 c_{n, k}\right)^{n-2 k+2} \iint_{Q_{\frac{5}{2}}(0,0)} \frac{1}{2}\left|\psi_{\lambda}^{(X, T)}\right|^{2} \cdot \sqrt{g_{\lambda}^{X}} \stackrel{\lambda \searrow 0}{\longrightarrow} 0,
\end{aligned}
$$

where in the second line we used the fact that $Q_{2 \lambda}(x, t) \subset Q_{\frac{5 \lambda}{2}}(X, T)$ for all $(x, t) \in$ $Q_{\lambda / 2}(X, T)$ and in the last line we used the fact that

$$
\left|\psi_{\lambda}^{(X, T)}\right|^{2}(y, s)=\lambda^{2 k}|\psi|^{2}\left(\vartheta_{X}(\lambda y), T+\lambda^{2} s\right) \stackrel{\lambda \searrow 0}{\longrightarrow} 0
$$

$\vartheta_{X}$ being a geodesic normal parametrisation of $B_{\text {inj }_{X}}(X)$, as well as the dominated convergence theorem. Therefore, there exists a $\lambda_{0} \leq i_{0}$ for which the $\varepsilon$-regularity condition

$$
\sup _{(x, t) \in Q_{\lambda_{0} / 2}(X, T)} I_{k}\left(u, g ; x, t, \frac{\lambda_{0}}{2 c_{n, k}}\right)<\varepsilon
$$

holds. Theorem 3.1 therefore gives us a necessary and sufficient condition for the local extensibility of $\{u(\cdot, t)\}$ up to time $t=T$.

A cursory inspection of the preceding remark shows that we may in fact extract a local regularity theorem based around parabolic cylinders rather than heat balls from Theorem 3.1. This is the content of the following corollary.

Corollary 3.4 There exist geometric constants $\varepsilon_{0}, C_{0}>0$ such that for any $R \leq i_{0}$ and Yang-Mills or harmonic map heat flow $u$ on $Q_{R}(X, T) \subset \Omega \times \mathbb{R}$, the implication 


$$
\frac{1}{R^{n-2 k+2}} \iint_{Q_{R}(X, T)} \frac{1}{2}|\psi|^{2}<\varepsilon_{0} \Rightarrow R^{2 k} \sup _{Q_{R / 10}(X, T)} \frac{1}{2}|\psi|^{2} \leq C_{0}
$$

holds.

Proof Let $\varepsilon_{0}=\frac{\varepsilon}{\gamma_{n, k} \cdot\left(5 c_{n, k}\right)^{n-2 k+2}}$, where $\varepsilon$ is as in Theorem 3.1 and $\gamma_{n, k}$ as in Lemma 2.10. We compute as in Remark 3.3 that

$$
\begin{aligned}
& \sup _{(x, t) \in Q_{\frac{2 R / 5}{2}}(X, T)} I_{k}\left(u, g ; x, t, \frac{2 R / 5}{2 c_{n, k}}\right) \\
& \leq \gamma_{n, k} \cdot\left(5 c_{n, k}\right)^{n-2 k+2} \cdot \frac{1}{R^{n-2 k+2}} \iint_{Q_{R}(X, T)} \frac{1}{2}|\psi|^{2}<\varepsilon
\end{aligned}
$$

so that we may apply Theorem 3.1 to obtain the desired estimate with $C_{0}=C$.

Remark 3.5 Arguing exactly as in Remark 3.3, we deduce a second criterion for local extensibility of a harmonic map or Yang-Mills heat from Corollary 3.4, this time in terms of parabolic cylinders: If $u$ is a harmonic map or Yang-Mills heat flow on $\Omega \times\left[0, T\left[\right.\right.$ and $X \in \Omega$, then there exists an $R>0$ with $Q_{R}(X, T) \subset \Omega \times[0, T[$ such that $\left.u\right|_{Q_{R}(X, T)}$ may be smoothly extended to all of $\overline{Q_{R}(X, T)}$ if and only if there exists an $\left.R_{0} \in\right] 0, i_{0}[$ such that

$$
\frac{1}{R_{0}^{n-2 k+2}} \iint_{Q_{R_{0}}(X, T)} \frac{1}{2}|\psi|^{2}<\varepsilon_{0}
$$

with $\varepsilon_{0}$ as in Corollary 3.4 .

\section{The Singular Set}

We shall now make use of the local regularity theorem to characterise the singular set at the maximal time of a smooth Yang-Mills or harmonic map heat flow and draw conclusions on its measure-theoretic size.

For $(X, T) \in \Omega \times \mathbb{R}$, set

$$
R_{0}(X, T)=\min \left\{i_{0}, \operatorname{dist}(X, \partial \Omega), \frac{\sqrt{T}}{2}\right\}
$$

and let $u$ be a harmonic map or Yang-Mills heat flow on $\Omega \times[0, T[$. We shall call $(X, T) \in \Omega \times \mathbb{R}$ a regular point if there exists an $R>0$ such that $\overline{Q_{R}(X, T)} \subset$ $\Omega \times[0, T]$ and $\left.u\right|_{Q_{R}(X, T)}$ may be smoothly extended to all of $\overline{Q_{R}(X, T)}$. The singular set of $u$ at time $T$ is then defined to be

$$
\mathcal{S}=\Omega \backslash\{X \in \Omega:(X, T) \text { is a regular point }\}
$$


Now, it follows from Remarks 3.3 and 3.5 that regular points are characterised by either of the $\varepsilon$-regularity conditions of Theorem 3.1 and Corollary 3.4. Therefore, we may write $\mathcal{S}$ variously as

$$
\begin{aligned}
\mathcal{S} & =\left\{X \in \Omega: \forall R<R_{0}(X, T) \exists(x, t) \in Q_{\frac{R}{2}}(X, T) \text { s.t. } I_{k}\left(u, g ; x, t, \frac{R}{2 c_{n, k}}\right) \geq \varepsilon\right\} \\
& =\left\{X \in \Omega: \forall R<R_{0}(X, T) \frac{1}{R^{n-2 k+2}} \iint_{Q_{R}(X, T)} \frac{1}{2}|\psi|^{2} \geq \varepsilon_{0}\right\},
\end{aligned}
$$

where $\varepsilon$ and $\varepsilon_{0}$ are as in Theorem 3.1 and Corollary 3.4, respectively. The following lemma tells us that we can actually measure $\mathcal{S}$.

Lemma 4.1 $\mathcal{S}$ is closed in $\Omega$.

Proof If $X \in \Omega \backslash \mathcal{S}$, then we may smoothly extend $\left.u\right|_{Q_{R}(X, T)}$ to all of $\overline{Q_{R}(X, T)} \subset$ $\Omega \times[0, T]$ for some $R>0$, but this implies that $\left.u\right|_{Q_{R / 2}(\tilde{X}, T)}$ may be extended to all of $\bar{Q}_{R / 2}(\tilde{X}, T) \subset \Omega \times[0, T]$ for all $\tilde{X} \in B_{R / 2}(X)$, i.e. $B_{R / 2}(X) \subset \Omega \backslash \mathcal{S}$ so that $\mathcal{S}$ is closed.

Knowing that $\mathcal{S}$ is measurable, we shall now estimate its $(n-2 k)$-dimensional Hausdorff measure (denoted by $\mathscr{H}^{n-2 k}$ ) under the assumption of summability of $|\psi|^{2}$.

Corollary 4.2 (Singular set estimate) Suppose $|\psi|^{2} \in L^{1}(\Omega \times[0, T[)$. Then for any $K \Subset \Omega$ and $\left.\delta_{0} \in\right] 0$, $\operatorname{dist}(K, \partial \Omega)[$, the estimate

$$
\mathscr{H}^{n-2 k}(\mathcal{S} \cap K) \leq \frac{5^{n-2 k}}{\varepsilon_{0}} \limsup _{t \nearrow T} \int_{B_{\delta_{0}}(\mathcal{S} \cap K)} \frac{1}{2}|\psi|^{2}(\cdot, t) d v o l_{g}
$$

holds, where $\varepsilon_{0}$ is as in Corollary 3.4.

Proof Fix $\delta_{1}>0$ and $0<\delta \ll \min \left\{\delta_{1}, \delta_{0}\right\}$, and cover $\mathcal{S} \cap K$ with the family of balls $\left\{B_{\delta}(x)\right\}_{x \in \mathcal{S} \cap K}$. By compactness and the Vitali covering lemma, we may pass to a finite pairwise disjoint subfamily $\left\{B_{\delta}\left(x_{i}\right)\right\}_{i \in I_{0}}$ such that $\mathcal{S} \cap K \subset \bigcup_{i \in I_{0}} B_{5 \delta}\left(x_{i}\right)$ so that the latter equality of (4.1) implies that

$$
\begin{aligned}
\sum_{i \in I_{0}}(5 \delta)^{n-2 k} & \leq \frac{5^{n-2 k}}{\varepsilon_{0}} \sum_{i \in I_{0}} \delta^{-2} \int_{T-\delta^{2}}^{T} \int_{B_{\delta}\left(x_{i}\right)} \frac{1}{2}|\psi|^{2} \\
& \leq \frac{5^{n-2 k}}{\varepsilon_{0}} \sup _{t \in] T-\delta_{1}^{2}, T[} \int_{B_{\delta_{0}}(\mathcal{S} \cap K)} \frac{1}{2}|\psi|^{2}(\cdot, t) .
\end{aligned}
$$

Passing to the limit $\delta \searrow 0$ then $\delta_{1} \searrow 0$ yields the desired estimate.

Remark 4.3 Note that the right-hand side of (4.2) is finite by virtue of Remark 2.11. Moreover, in contrast to the case of the corresponding statement in the static case on 
the singular set of stationary harmonic and energy-minimising maps (cf. [32, Corollary 2.7] and [5, Sect. VII]), it is not possible in general to take a further limit $\delta_{0} \searrow 0$ in (4.2). However, if we assume that $\psi$ satisfies the energy continuity hypothesis

$$
\lim _{t \nearrow T} \int_{M} \frac{1}{2}|\psi|^{2}(\cdot, t) \cdot \varphi=\int_{M} f \cdot \varphi
$$

for some $f \in L_{\text {loc }}^{1}\left(M,\left[0, \infty[)\right.\right.$ and all $\varphi \in C_{0}^{\infty}(M)$, then we may choose for each small $\delta_{0}>0$ a test function $\varphi: M \rightarrow[0,1]$ with supp $\varphi \subset B_{2 \delta_{0}}(\mathcal{S} \cap K)$ and $\left.\varphi\right|_{B_{\delta_{0}}(\mathcal{S} \cap K)} \equiv 1$ so that

$$
0 \leq \limsup _{t \nearrow T} \int_{B_{\delta_{0}}(\mathcal{S} \cap K)} \frac{1}{2}|\psi|^{2}(\cdot, t) \leq \lim _{t \nearrow T} \int_{M} \frac{1}{2}|\psi|^{2}(\cdot, t) \cdot \varphi \leq \int_{B_{2 \delta_{0}}(\mathcal{S} \cap K)} f,
$$

which then implies after taking the limit $\delta_{0} \searrow 0$, by virtue of the fact that $\mathcal{S} \cap K$ is of Lebesgue measure zero, that $\mathscr{H}^{n-2 k}(\mathcal{S} \cap K)=0$ for any $K \Subset \Omega$. This should be compared with the case of the mean curvature flow, where a similar continuity hypothesis is necessary to deduce a measure-zero singular set (see [13, Chap. 5]).

Remark 4.4 The definition we have given of the singular set $\mathcal{S}$ applies just as well if $u$ is defined on all of $M \times[0, T$, i.e. we say that $(X, T) \in M \times \mathbb{R}$ is a regular point if there exists an $R>0$ such that $\left.u\right|_{Q_{R}(X, T)}$ may be smoothly extended to all of $\overline{Q_{R}(X, T)}$ and let $\mathcal{S}=M \backslash\{X \in M:(X, T)$ is a regular point $\}$. In this case, equalities (4.1) hold provided $\mathcal{S}$ is replaced with $\mathcal{S} \cap \Omega$, and $\mathcal{S}$ may be shown to be closed just as in Lemma 4.1. Moreover, Corollary 4.2 continues to hold. For both of the corresponding statements, it should be borne in mind that the geometric constants $\varepsilon$ and $\varepsilon_{0}$ depend on the geometry of $\Omega$.

\section{Rapidly Forming Singularities}

We now turn our attention to rapidly forming (or type-I) singularities of the harmonic map and Yang-Mills heat flows. Analogues of the results of this section have been established in the case of a compact base manifold $M$ by Grayson and Hamilton [20] for the harmonic map heat flow and Weinkove [41] for the Yang-Mills heat flow, and examples of heat flows admitting such singularities may be found in [18], [19] and [41].

A Yang-Mills or harmonic map heat flow $u$ on $\Omega \times[0, T$ [ is said to have a rapidly forming singularity at $(X, T)$ if its fundamental form $\psi$ satisfies the scale-invariant estimate

$$
\sup _{(x, t) \in Q_{R}(X, T)}(T-t)^{k} \frac{1}{2}|\psi|^{2}(x, t) \leq C_{0}
$$

for some $C_{0}>0$ and $R>0$ with $\left.Q_{R}(X, T) \subset \Omega \times\right] 0, T$. We first show that rapidly forming singularities admit smooth blow-ups. 
Lemma 5.1 (Existence of blow-ups) Suppose $u$ is a Yang-Mills or harmonic map heat flow defined on $\Omega \times[0, T$ [ and has a rapidly forming singularity at $(X, T) \in \Omega \times \mathbb{R}$. Then there exists a Yang-Mills (resp. harmonic map) heat flow $u_{\infty}$ on $\left.\mathbb{R}^{n} \times\right]-\infty, 0[$ such that $u_{r}^{(X, T)} \stackrel{r \searrow 0}{\longrightarrow} u_{\infty}$ subsequentially and locally uniformly in $C^{\infty}$ on $\left.\mathbb{R}^{n} \times\right]-$ $\infty, 0[$ (modulo gauge).

Proof We shall assume without loss of generality that $R<i_{0}$. Fix $\left(x_{0}, t_{0}\right) \in$ $Q_{\frac{R}{2}}(X, T)$. We have that $Q_{\widetilde{R}}\left(x_{0}, t_{0}\right) \subset Q_{R}(X, T)$ with $\widetilde{R}:=\sqrt{T-t_{0}}$. Moreover, the estimate (5.1) implies that

$$
\sup _{Q_{\widetilde{R}}\left(x_{0}, t_{0}\right)} \frac{1}{2}|\psi|^{2} \leq \frac{C_{0}}{\widetilde{R}^{2 k}} .
$$

Lemma 2.1 then implies that for each $i \in \mathbb{N}$,

$$
\sup _{Q_{\frac{\widetilde{R}}{2}}\left(x_{0}, t_{0}\right)} \frac{1}{2}\left|\nabla^{i} \psi\right|^{2} \leq \frac{C_{i}}{\widetilde{R}^{2(k+i)}},
$$

where $C_{i}$ depends on $C_{0}$ and the geometries of $\Omega$ and the target manifold of the respective flow (as in Lemma 2.1). Therefore, we have the estimate

$$
\sup _{(x, t) \in Q_{\frac{R}{2}}(X, T)}(T-t)^{k+i} \frac{1}{2}\left|\nabla^{i} \psi\right|^{2}(x, t) \leq C_{i} .
$$

Using the compactness of the target manifold of the harmonic map heat flow and arguing as in Lemma 2.4 for the Yang-Mills heat flow, choosing a suitable fixed connection as in Remark 2.5, we obtain bounds

$$
\sup _{Q_{\theta R}(X, T)}(T-t)^{k+i-1} \frac{1}{2}\left|\nabla^{i} u\right|^{2} \leq \bar{C}_{i-1}
$$

for $i \in \mathbb{N} \cup\{0\},\left\{\bar{C}_{i}\right\}_{i=-1}^{\infty}$ depending on the $\left\{C_{i}\right\}_{i=0}^{\infty}$, and $\left.\left.\theta \in\right] 0, \frac{1}{4}\right]$. After rescaling, these estimates imply that for any $r>0$,

$$
\sup _{(x, t) \in Q_{\frac{\theta R}{r}}(0,0)}(-t)^{k+i-1} \frac{1}{2}\left|\nabla^{i} u_{r}^{(X, T)}\right|^{2}(x, t) \leq \widetilde{C}_{i-1}
$$

for constants $\left\{\widetilde{C}_{i}\right\}_{i=-1}^{\infty}$ depending on $\left\{\bar{C}_{i}\right\}_{i=-1}^{\infty}$. Thus, considering a sequence $\left\{u_{i}=\right.$ $\left.u_{r_{i}}^{(X, T)}\right\}_{i \in \mathbb{N}}$ of rescalings of $u$ with $r_{i} \searrow 0$ and using (5.2) for each compact subset of $\left.\mathbb{R}^{n} \times\right]-\infty, 0\left[\right.$, we obtain, for large enough $i$, bounds on $u_{i}$ and all of its derivatives. Therefore, by the Arzelà-Ascoli theorem, we may pass to a subsequence converging locally uniformly in $C^{\infty}$ to a smooth solution $\left.u_{\infty}: \mathbb{R}^{n} \times\right]-\infty, 0\left[\rightarrow V \otimes \Lambda^{k-1} T^{*} \mathbb{R}^{n}\right.$ to the corresponding flow equation on $\left(\mathbb{R}^{n}, g_{\delta}\right)$. 
The following corollary establishes that smooth blow-ups of rapidly forming singularities are in fact self-similar and gives us a strictly positive lower bound on their heat ball energies; the latter guarantees that such blow-ups are nontrivial.

Corollary 5.2 (Self-similarity and nontriviality of blow-ups) Let $\left\{u_{r_{i}}^{(X, T)}\right\}_{i \in \mathbb{N}}$ be a sequence of rescalings ( $r_{i} \searrow 0$ ) of a Yang-Mills or harmonic map heat flow with rapidly forming singularity at $(X, T) \in \Omega \times \mathbb{R}$. Moreover, suppose $u_{r_{i}}^{(X, T)} \stackrel{i \rightarrow \infty}{\longrightarrow} u_{\infty}$ locally uniformly in $C^{\infty}$ on $\left.\mathbb{R}^{n} \times\right]-\infty, 0\left[\right.$. Then $u_{\infty}$ is self-similar. Moreover, $u_{\infty}$ satisfies the estimate

$$
I_{k}\left(u_{\infty}, g_{\delta} ; 0,0, R\right)=\frac{1}{R^{n-2 k}} \iint_{E_{R}^{n-2 k}(0,0)} \frac{1}{2}\left|\psi_{\infty}\right|^{2}(x, t) \cdot\left(\frac{n-2 k}{-2 t}\right) d x d t \geq \varepsilon
$$

for all $R>0$, where $\psi_{\infty}$ is the fundamental form corresponding to $u_{\infty}$ and $\varepsilon$ is as in Theorem 3.1.

Proof We first establish self-similarity. By virtue of the higher-order estimates derived in Lemma 5.1, the monotone quantity (2.19) is finite on $u$ for sufficiently small $R$. Therefore, we may take its limit as $R \searrow 0$ and freely apply scale invariance (Remark 2.8) so that for any $r>0$,

$$
\begin{aligned}
& \lim _{R \searrow 0} I_{k}(u, g ; X, T, R)=\lim _{i \rightarrow \infty} I_{k}\left(u, g ; X, T, r_{i} r\right) \\
& \quad=\lim _{i \rightarrow \infty} \frac{1}{r^{n-2 k}} \iint_{E_{r}^{n-2 k}(0,0)} e^{\xi_{k}\left(-\lambda_{i}^{2} s\right)} e_{k}\left[u_{r_{i}}^{(X, T)}, g_{r_{i}}^{X}\right]_{(0,0)}(y, s) \sqrt{g_{r_{i}}^{X}}(y, s) \mathrm{d} y \mathrm{~d} s \\
& \quad=\frac{1}{r^{n-2 k}} \iint_{E_{r}^{n-2 k}(0,0)} e_{k}\left[u_{\infty}, g_{\delta}\right]_{(0,0)}(y, s) \mathrm{d} y \mathrm{~d} s=I_{k}\left(u_{\infty}, g_{\delta} ; 0,0, r\right) .
\end{aligned}
$$

Thus, this last quantity is independent of $r$ and so, by Theorem $2.6, u_{\infty}$ must be self-similar.

We now turn our attention to the estimate (5.3). We first fix a sequence $\lambda_{i} \searrow 0$. Since $X \in \mathcal{S}$, the first equality of (4.1) implies that there exists a sequence $\left(x_{i}, t_{i}\right) \in$ $Q_{c_{n, k} \lambda_{i} r}(X, T)$ such that for sufficiently large $i$ and small fixed $r>0$, we have

$$
I_{k}\left(u, g ; x_{i}, t_{i}, \lambda_{i} r\right) \geq \varepsilon
$$

Using monotonicity and translation-invariance (Theorem 2.6 and Remark 2.8), we arrive at the inequality

$$
\frac{1}{r^{n-2 k}} \iint_{E_{r}^{n-2 k}(0,0)} e_{k}\left[u_{1}^{\left(x_{i}, t_{i}\right)}, g_{1}^{x_{i}}\right]_{(0,0)}(y, s) e^{\xi_{k}(-s)} \sqrt{g_{1}^{x_{i}}}(y) \mathrm{d} y \mathrm{~d} s \geq \varepsilon .
$$

Taking the limit $i \rightarrow \infty$, noting that $\left(x_{i}, t_{i}\right) \stackrel{i \rightarrow \infty}{\longrightarrow}(X, T)$ and using the higher-order estimates of Lemma 5.1, we obtain the inequality

$$
I_{k}(u, g ; X, T, r) \geq \varepsilon
$$


We now let $r=\lambda_{i}$, use scale invariance and pass to the limit $i \rightarrow \infty$ to obtain (5.3) with $R=1$, but the self-similarity of $u_{\infty}$ implies that (5.3) holds for all $R>0$.

Remark 5.3 Since the blow-up $u_{\infty}$ in Corollary 5.2 is self-similar, it follows from [14, Proposition 1.5] for the harmonic map heat flow and [1, Proposition 2.2] for the Yang-Mills heat flow that

$$
\int_{\mathbb{R}^{n}} \frac{1}{2}\left|\psi_{\infty}\right|^{2} \cdot \Phi_{(0,0)}^{k}(x, t) \mathrm{d} x=I_{k}\left(u_{\infty}, g_{\delta} ; 0,0, R\right) \geq \varepsilon
$$

for all $R>0$ and $t<0$, where $\Phi_{(0,0)}^{k}(x, t)=\frac{1}{(-4 \pi t)^{\frac{n-2 k}{2}}} \exp \left(\frac{|x|^{2}}{4 t}\right)$ and $\varepsilon>0$ is as in Theorem 3.1. Moreover, we may use (5.1) to establish the existence of a $\rho>0$ depending only on $n, k, C_{0}$ and $\varepsilon$ such that

$$
\int_{\mathbb{R}^{n} \backslash B_{\rho \sqrt{-t}}(0)} \frac{1}{2}\left|\psi_{\infty}\right|^{2} \cdot \Phi_{(0,0)}^{k}(x, t) \mathrm{d} x \leq C_{0} \cdot(4 \pi)^{\frac{2 k-n}{2}} \int_{\mathbb{R}^{n} \backslash B_{\rho}(0)} \exp \left(-\frac{1}{4}|z|^{2}\right) \mathrm{d} z<\frac{\varepsilon}{2}
$$

so that in fact, for this $\rho>0$, we have the estimate

$$
\int_{B_{\rho \sqrt{-t}}(0)} \frac{1}{2}\left|\psi_{\infty}\right|^{2} \cdot \Phi_{(0,0)}^{k}(x, t) \mathrm{d} x \geq \frac{\varepsilon}{2}
$$

for all $t<0$. We have thus recovered the results of [20, Sect. 5] and [41, Sect. 3] established in the case of blow-ups at rapidly forming singularities on compact $M$. We note that the constant $\varepsilon>0$ employed there additionally depends on the initial energy of the flow.

Acknowledgements The author would like to thank Matthew Cooper, Klaus Ecker and Tobias Marxen for helpful discussions regarding an earlier version of this manuscript. The author gratefully acknowledges financial support from the Freie Universität Berlin. Part of this work was completed while the author was employed at the Leibniz Universität Hannover.

Funding Open Access funding enabled and organized by Projekt DEAL.

Open Access This article is licensed under a Creative Commons Attribution 4.0 International License, which permits use, sharing, adaptation, distribution and reproduction in any medium or format, as long as you give appropriate credit to the original author(s) and the source, provide a link to the Creative Commons licence, and indicate if changes were made. The images or other third party material in this article are included in the article's Creative Commons licence, unless indicated otherwise in a credit line to the material. If material is not included in the article's Creative Commons licence and your intended use is not permitted by statutory regulation or exceeds the permitted use, you will need to obtain permission directly from the copyright holder. To view a copy of this licence, visit http://creativecommons.org/licenses/by/4.0/.

\section{References}

1. Afuni, A.: Local monotonicity for the Yang-Mills-Higgs flow. Calc. Var. 555(1), 1-14 (2016)

2. Afuni, A.: Energy identities and monotonicity for evolving $k$-forms on moving Riemannian spaces. J. Evol. Equ. 18(2), 549-560 (2018) 
3. Afuni, A.: Heat ball formulæ for $k$-forms on evolving manifolds. Adv. Calc. Var. 12(2), 135-156 (2019)

4. Atiyah, M.F., Bott, R.: The Yang-Mills equations over Riemann surfaces. Philos. Trans. R. Soc. Lond. Ser. A 308(1505), 523-615 (1983)

5. Bethuel, F.: On the singular set of stationary harmonic maps. Manuscr. Math. 78(4), 417-443 (1993)

6. Booss, B., Bleecker, DD.: Topology and analysis. The Atiyah-Singer index formula and gauge- theoretic physics. Transl. from the German by D. D. Bleecker and A. Mader. Springer, Berlin (1985)

7. Chang, K.-C., Ding, W.Y., Ye, R.: Finite-time blow-up of the heat flow of harmonic maps from surfaces. J. Differ. Geom. 36(2), 507-515 (1992)

8. Chen, Y., Shen, C.: Monotonicity formula and small action regularity for Yang-Mills flows in higher dimensions. Calc. Var. Partial Differ. Equ. 2(4), 389-403 (1994)

9. Chen, Y., Shen, C., Zhou, Q.: Asymptotic behavior of Yang-Mills flow in higher dimensions. In: Differential Geometry and Related Topics, pp. 16-38. World Scientific (2002)

10. Coron, J.-M., Ghidaglia, J.-M.: Explosion en temps fini pour le flot des applications harmoniques. C. R. Acad. Sci. Paris Sér. I Math. 308(12), 339-344 (1989)

11. Donaldson, S.: Anti self-dual Yang Mills connections over complex algebraic surfaces and stable vector bundles. Proc. Lond. Math. Soc. 3(50), 1-26 (1985)

12. Douglis, A., Nirenberg, L.: Interior estimates for elliptic systems of partial differential equations. Commun. Pure Appl. Math. 8, 503-538 (1955)

13. Ecker, K.: Regularity Theory for Mean Curvature Flow. Progress in Nonlinear Differential Equations and their Applications, vol. 57. Birkhäuser, Boston (2004)

14. Ecker, K.: Local monotonicity formulas for some nonlinear diffusion equations. Calc. Var. Partial Differ. Equ. 23(1), 67-81 (2005)

15. Ecker, K., Huisken, G.: Mean curvature evolution of entire graphs. Ann. Math. 130(3), 453-471 (1989)

16. Eells Jr., J., Sampson, J.H.: Harmonic mappings of Riemannian manifolds. Am. J. Math. 86, 109-160 (1964)

17. Enders, J., Muller, R., Topping, P.M.: On type-I singularities in Ricci flow. Commun. Anal. Geom. 19(55), 905-922 (2011)

18. Fan, H.: Existence of the self-similar solutions in the heat flow of harmonic maps. Sci. China Ser. A 42(2), 113-132 (1999)

19. Gastel, A.: Singularities of first kind in the harmonic map and Yang-Mills heat flows. Mathematische Zeitschrift 242(1), 47-62 (2002)

20. Grayson, M., Hamilton, R.S.: The formation of singularities in the harmonic map heat flow. Commun. Anal. Geom. 4(4), 525-546 (1996)

21. Grotowski, J.F.: Finite time blow-up for the Yang-Mills heat flow in higher dimensions. Math. Z. 237(2), 321-333 (2001)

22. Hamilton, R.S.: Monotonicity formulas for parabolic flows on manifolds. Commun. Anal. Geom. 1(1), 127-137 (1993)

23. Hong, M.-C., Tian, G.: Asymptotical behaviour of the Yang-Mills flow and singular Yang-Mills connections. Math. Ann. 330(3), 441-472 (2004)

24. Morrey, C.B. Jr.: Multiple integrals in the calculus of variations. Die Grundlehren der mathematischen Wissenschaften, vol. 130, p. 506. Springer, New York (1966)

25. Naito, H.: Finite time blowing-up for the Yang-Mills gradient flow in higher dimensions. Hokkaido Math. J. 23(3), 451-464 (1994)

26. Nakajima, H.: Compactness of the moduli space of Yang-Mills connections in higher dimensions. J. Math. Soc. Jpn. 40(3), 383-392 (1988)

27. Ni, L.: Mean value theorems on manifolds. Asian J. Math. 11(2), 277-304 (2007)

28. Petersen, P.: Riemannian Geometry. Graduate Texts in Mathematics, vol. 171, 2nd edn. Springer, New York (2006)

29. Poor, W.A.: Differential Geometric Structures. McGraw-Hill Book Company Inc., New York (1981)

30. Price, P.: A monotonicity formula for Yang-Mills fields. Manuscr. Math. 43, 131-166 (1983)

31. Råde, J.: Singular Yang-Mills connections. In: Journées Équations aux Dérivées Partielles (Saint-Jeande-Monts, 1995), pp. Exp. No. VIII, 15. École Polytech., Palaiseau (1995)

32. Schoen, R., Uhlenbeck, K.: A regularity theory for harmonic maps. J. Differ. Geom. 17, 307-335 (1982)

33. Shi, W.-X.: Deforming the metric on complete Riemannian manifolds. J. Differ. Geom. 30(1), 223-301 (1989) 
34. Struwe, M.: On the evolution of harmonic mappings of riemannian surfaces. Commentarii Mathematici Helvetici 60(1), 558-581 (1985)

35. Struwe, M.: On the evolution of harmonic maps in higher dimensions. J. Differ. Geom. 28(3), 485-502 (1988)

36. Struwe, M.: The Yang-Mills flow in four dimensions. Calc. Var. Partial Differ. Equ. 2(2), 123-150 (1994)

37. Synge, J.L., Schild, A.: Tensor calculus. Repr. from corr. ed. 1969 (Univ. of Toronto Press) (1978)

38. Uhlenbeck, K.: Removable singularities in Yang-Mills fields. Commun. Math. Phys. 83(1), 11-29 (1982)

39. Waldron, A.: Long-time existence for Yang-Mills flow. Invent. Math. 217, 1069-1147 (2019)

40. Warner, F.W.: Foundations of differentiable manifolds and Lie groups, Graduate Texts in Mathematics, vol. 94. Springer, New York (1983). Corrected reprint of the 1971 edition

41. Weinkove, B.: Singularity formation in the Yang-Mills flow. Calc. Var. Partial Differ. Equ. 19(2), 211-220 (2004)

42. White, B.: A local regularity theorem for mean curvature flow. Ann. Math. 161(3), 1487-1519 (2005)

Publisher's Note Springer Nature remains neutral with regard to jurisdictional claims in published maps and institutional affiliations. 